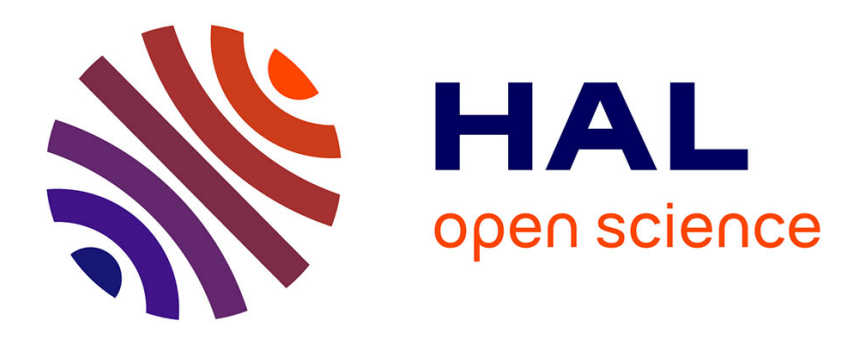

\title{
Géométrie des nombres adélique et lemmes de Siegel généralisés \\ Éric Gaudron
}

\section{To cite this version:}

Éric Gaudron. Géométrie des nombres adélique et lemmes de Siegel généralisés. Manuscripta mathematica, 2009, 130 (2), pp.159-182. 10.1007/s00229-009-0282-3 . hal-02175092

\section{HAL Id: hal-02175092 \\ https://hal.science/hal-02175092}

Submitted on 5 Jul 2019

HAL is a multi-disciplinary open access archive for the deposit and dissemination of scientific research documents, whether they are published or not. The documents may come from teaching and research institutions in France or abroad, or from public or private research centers.
L'archive ouverte pluridisciplinaire HAL, est destinée au dépôt et à la diffusion de documents scientifiques de niveau recherche, publiés ou non, émanant des établissements d'enseignement et de recherche français ou étrangers, des laboratoires publics ou privés. 


\title{
GÉOMÉTRIE DES NOMBRES ADÉLIQUE ET LEMMES DE SIEGEL GÉNÉRALISÉS
}

\author{
ÉRIC GAUDRON
}

\begin{abstract}
RÉsumé. Ce texte présente plusieurs énoncés qui assurent l'existence d'un vecteur non nul de petite hauteur dans un espace vectoriel de dimension finie sur un corps de nombres et qui évite un nombre fini de sous-espaces donnés au préalable (lemmes de Siegel avec contraintes). Les démonstrations reposent sur des résultats de géométrie des nombres adélique, dont, en particulier, une variante adélique d'un théorème de Henk sur la fonction de dénombrement des points d'un réseau dans un corps convexe symétrique.
\end{abstract}

Abstract. A Siegel's lemma provides an explicit upper bound for a non-zero vector of minimal height in a finite dimensional vector spaces over a number field. This article explains how to obtain Siegel's lemmas for which the minimal vectors do not belong to a finite union of vector subspaces (Siegel's lemmas with conditions). The proofs mix classical results of adelic geometry of numbers and an adelic variant of a theorem of Henk about the number of lattice points of a centrally symmetric convex body in terms of the successive minima of the body.

\section{Contact details of the author :}

Éric GAUDRON

凶niversité Grenoble I, Institut Fourier.

UMR 5582, BP 74

38402 Saint-Martin-d'Hères Cedex, France.

Courriel : Eric.Gaudron@ujf-grenoble.fr

ऽ (33) 0476514572

Page internet : http ://www-fourier.ujf-grenoble.fr/ gaudron

MSC 2000 : 11J68 (11H06,11G50,14G40)

Mots clefs : Lemme de Siegel, fibré vectoriel adélique, géométrie des nombres adélique, minima successifs, théorème de van der Corput, théorème de Blichfeldt, théorème de Henk, décompte de vecteurs algébriques dans une boule adélique.

Date: Mercredi 13 mai 2009. 


\section{INTRODUCTION}

En théorie des nombres transcendants, un lemme de Siegel est un énoncé qui garantit l'existence d'un vecteur non nul et de « petite » hauteur dans un espace vectoriel donné. Dans ce texte, nous présentons une généralisation qui, outre cette condition, contraint le petit vecteur à n'être dans aucun d'un nombre fini de sousespaces, fixés au préalable. Notre étude s'inscrit dans la suite des travaux de L. Fukshansky qui a examiné la question dans deux articles $[4,5]$. Toutefois notre approche est un peu différente et elle conduit à de meilleurs résultats.

Soit $k$ un corps de nombres. Dans l'énoncé qui va suivre, on se donne un fibré vectoriel adélique pur $\bar{E}$ sur Spec $k$. Il s'agit d'un $k$-espace vectoriel $E$ de dimension finie muni d'une collection de normes $\|\cdot\|_{\bar{E}}, v$ aux places $v$ de $k$, soumises à quelques conditions techniques (voir définition 2.1). Si $\|\cdot\|_{\bar{E}, v}$ est une norme hermitienne en toutes les places $v$ archimédiennes, le fibré vectoriel adélique $\bar{E}$ est dit hermitien. Par exemple $k^{n}$ muni des normes $\left(\sum_{i}\left|x_{i}\right|_{v}^{2}\right)^{1 / 2}$ si $v$ est archimédienne et $\max _{i}\left\{\left|x_{i}\right|_{v}\right\}$ si $v$ est ultramétrique est un fibré adélique hermitien $\operatorname{sur} \operatorname{Spec} k$, noté $\left(k^{n},|\cdot|_{2}\right)$. À un tel objet sont associées plusieurs notions de hauteurs :

(i) Celle d'un vecteur $x$ de $E$, définie comme le nombre réel $H_{\bar{E}}(x):=\prod_{v}\|x\|_{\bar{E}, v}^{n_{v}}$ où $v$ parcourt l'ensemble des places de $k$ et $n_{v}:=\left[k_{v}: \mathbf{Q}_{v}\right]$ est le degré local.

(ii) Celle d'un sous-espace $F$ de $E$, définie comme le nombre réel $H(\bar{F}):=$ $H \overline{\bigwedge^{m} E}\left(f_{1} \wedge \cdots \wedge f_{m}\right)$ où $\left(f_{1}, \ldots, f_{m}\right)$ est une $k$-base de $F$ et $\overline{\wedge^{m} E}$ est le produit extérieur d'ordre $m$ de $E$, muni de la structure hermitienne quotient déduite de celle de $\bar{E}$ (si $F=\{0\}$ alors on pose $H(\bar{F})=1$ ).

Soulignons que ces hauteurs ne sont pas normalisées par le degré du corps de nombres. Enfin, si $F \subseteq E$ est un sous-espace vectoriel de $E$, nous désignons par $\Lambda_{1}(\bar{F})$ la plus petite hauteur $H_{\bar{E}}(f)$ lorsque $f$ parcourt $F \backslash\{0\}$ (par convention $\left.\Lambda_{1}(\overline{\{0\}})=1\right)$. Toutes ces notions sont précisées au $\S 2$.

Dans ce cadre nous avons le résultat suivant.

Théorème 1.1. Soit $k$ un corps de nombres de degré $D$ et de discriminant $D_{k}$. Soit $\bar{E}$ un fibré adélique hermitien sur $\operatorname{Spec} k$, de dimension $n \geq 1$. Soit $M \in \mathbf{N} \backslash$ $\{0\}$. Soit $F, E_{1}, \ldots, E_{M}$ des sous-espaces vectoriels de $E$ de dimensions respectives $m, n_{1}, \ldots, n_{M}$. Posons

$$
H:=(41 m)^{D / 2}\left|D_{k}\right|^{1 / 2}(M H(\bar{F}))^{1 / m} .
$$

Supposons que $\max \left\{n_{1}, \ldots, n_{M}\right\}<m$. Alors il existe $x \in F \backslash \bigcup_{i=1}^{M} E_{i}$ tel que

$$
H_{\bar{E}}(x) \leq H \max _{1 \leq i \leq M}\left\{1,\left(\frac{H^{n_{i}}}{H\left(\overline{E_{i}}\right)}\right)^{\frac{1}{m-n_{i}}},\left(\frac{H}{\Lambda_{1}\left(\overline{E_{i}}\right)}\right)^{\frac{n_{i}-1}{m-n_{i}+1}}\right\}
$$

(les trois termes entre accolades sont remplacés par 1 lorsque $n_{i}$ est nul).

Le cas $M=1$ et $E_{1}=\{0\}$ est le cas classique du lemme de Siegel et le théorème 1.1 donne la borne $H_{\bar{E}}(x) \leq(41 m)^{D / 2}\left|D_{k}\right|^{1 / 2} H(\bar{F})^{1 / m}$ qui est celle de Bombieri \& Vaaler [2], à la constante $\sqrt{41}^{D}$ près. Ce théorème tient compte de la hauteur et du premier minimum des $E_{i}$. Mais l'on peut obtenir aisément une estimation qui ne dépende pas des $E_{i}$, comme me l'a expliqué Gaël Rémond (voir $\S 3$ et le corollaire 5.2). Par ailleurs, l'hypothèse « $\bar{E}$ hermitien »n'est pas importante. Elle peut être supprimée à condition de remplacer le terme $m^{D / 2}$ qui est dans $H$ par $m^{D}$, et de revoir la définition de $H(\bar{F})$ (voir le $\S 2.2$ et le théorème général 4.1 ). On vérifie aisément qu'avec le fibré adélique hermitien standard $\bar{E}=\left(k^{n},|\cdot|_{2}\right)$ tous les termes $\Lambda_{1}\left(\overline{E_{i}}\right), H(\bar{F}), H\left(\overline{E_{i}}\right)$ sont supérieurs à 1 . De cette observation et du théorème 1.1 l'on déduit l'existence d'une fonction $c=c(n, m, M, D)>0$ et d'un 
vecteur $x \in F \backslash \bigcup_{i=1}^{M} E_{i}$ tels que $H_{\left(k^{n},||_{2}\right)}(x) \leq c\left(\left|D_{k}\right|^{m / 2} H(\bar{F})\right)^{1 /(m-s)}$ où $s:=$ $\max \left\{n_{1}, \ldots, n_{M}\right\}$. $\hat{A}$ titre de comparaison, le théorème 3.1 de [5] donne la borne $H_{\left(k^{n},||_{2}\right)}(x) \leq c\left|D_{k}\right|^{m / 2} H(\bar{F})$ (voir aussi le corollaire 5.2). Le gain est particulièrement important lorsque tous les $E_{i}$ sont des droites. En effet, si $e_{1}, \ldots, e_{M} \in E \backslash\{0\}$, le théorème 1.1 assure l'existence de $x \in F \backslash \bigcup_{i=1}^{M} k . e_{i}$ tel que

$$
H_{\bar{E}}(x) \leq H \max _{1 \leq i \leq M}\left\{1, \frac{H}{H_{\bar{E}}\left(e_{i}\right)}\right\}^{\frac{1}{m-1}} .
$$

La démonstration du théorème 1.1 repose sur un principe très simple. Étant donné un idèle $r=\left(r_{v}\right)_{v} \in k_{\mathbf{A}}^{\times}$, l'existence d'un vecteur $x \in F \backslash\{0\}$, de hauteur plus petite que le module $|r|_{\mathbf{A}}$, est garantie dès lors que l'on prend $x \neq 0$ dans l'intersection de $F$ et de la boule adélique $\mathbf{B}_{\bar{E}}(0, r)=\left\{x=\left(x_{v}\right)_{v} \in E \otimes_{k} k_{\mathbf{A}} ; \forall v,\left\|x_{v}\right\|_{\bar{E}, v} \leq\left|r_{v}\right|_{v}\right\}$ de rayon $r$. Et si l'on veut que $x \notin \bigcup_{i=1}^{M} E_{i}$, il suffit de choisir $r$ de sorte que

$$
\sum_{i=1}^{M} \operatorname{card}\left(E_{i} \cap \mathbf{B}_{\bar{E}}(0, r)\right)<\operatorname{card}\left(F \cap \mathbf{B}_{\bar{E}}(0, r)\right) .
$$

Ainsi tout le travail consiste à encadrer les cardinaux qui interviennent ici. Il s'agit donc d'une variante adélique du problème classique de géométrie des nombres de l'évaluation du nombre de points d'un réseau $(F)$ dans un corps convexe $\left(\mathbf{B}_{\bar{E}}(0, r)\right)$. Nous établissons une minoration du cardinal de $F \cap \mathbf{B}_{\bar{E}}(0, r)$, analogue à l'inégalité de van der Corput dans le cas classique. Et pour la majoration nous nous appuyons sur un théorème de Henk qui fait intervenir les minima successifs d'un réseau [7]. L'exploitation de ces résultats marque la différence d'avec les travaux de Fukshansky $[4,5]$.

\section{TABLE DES MATIÈRES}

1. Introduction

2. Géométrie des nombres adélique

2.1. Fibrés vectoriels adéliques

2.2. Hauteurs

2.3. Inégalité de van der Corput adélique $\quad 7$

2.4. Minima successifs

2.5. Version adélique d'un théorème de Henk 12

3. Lemme de Siegel uniforme 14

4. Le théorème principal et sa démonstration 15

5. Variantes du théorème principal 16

6. Liens avec la géométrie des nombres classique 18

$\begin{array}{ll}\text { Remerciements } & 20\end{array}$

Références $\quad 20$

\section{GÉométrie des nombres AdÉLIQUe}

Dans ce paragraphe, nous traduisons quelques résultats de géométrie des nombres dans le langage des fibrés vectoriels adéliques.

2.1. Fibrés vectoriels adéliques. Étant donné une place $v$ d'un corps global $k$, on note $\mathbf{C}_{v}$ la complétion d'une clôture algébrique de $k_{v}$. On note aussi $k_{\mathbf{A}}$ l'anneau des adèles de $k$ et $k_{\mathbf{A}}^{\times}$le groupe des idèles de $k$. On pose $\delta:=0$ si $k$ est un corps de fonctions et $\delta:=1$ si $k$ est un corps de nombres. En une place $v$ de $k$ est choisie sur le complété $k_{v}$ une valeur absolue $|\cdot|_{v}$ telle que, si l'on note $n_{v}$ le degré local de $k_{v}$ 
sur $\mathbf{R}$ ( $v$ archimédienne), $\mathbf{Q}_{p_{v}}(\delta=1$ et $v$ ultramétrique de caractéristique résiduelle $\left.p_{v}\right)$ ou $\operatorname{sur}\left(\mathbf{F}_{p}(T)\right)_{v}(\delta=0$ et $p$ est la caractéristique de $k)$, alors $x \in k_{v} \mapsto|x|_{v}^{n_{v}}$ est le module de Haar normalisé en la place $v$. La formule du produit s'écrit alors

$$
\forall x \in k \backslash\{0\}, \quad \prod_{v \text { place de } k}|x|_{v}^{n_{v}}=1
$$

(voir [6], p. 25, pour plus de détails). Dans tout ce texte, la lettre $D$ désigne le degré de $k$ sur $\mathbf{Q}$ ou $\mathbf{F}_{p}(T)$ selon que $\delta=1$ ou 0 .

Définition 2.1. Soit $n \in \mathbf{N}$. Un fibré vectoriel adélique $\bar{E}=\left(E,\left(\|\cdot\|_{\bar{E}, v}\right)_{v}\right)$ sur Spec $k$, de dimension $n$, est la donnée d'un $k$-espace vectoriel $E$ de dimension $n$ et d'une famille de normes $\|\cdot\|_{\bar{E}, v}$ sur $E \otimes_{k} \mathbf{C}_{v}$, aux places $v$ de $k$, qui satisfont aux conditions suivantes :

1) Il existe une $k$-base $\left(e_{1}, \ldots, e_{n}\right)$ de $E$ telle que, pour toute place $v$ ultramétrique en dehors d'un nombre fini, la norme sur $E \otimes_{k} \mathbf{C}_{v}$ est donnée par

$$
\forall\left(x_{1}, \ldots, x_{n}\right) \in \mathbf{C}_{v}^{n}, \quad\left\|\sum_{i=1}^{n} x_{i} e_{i}\right\|_{\bar{E}, v}=\max _{1 \leq i \leq n}\left\{\left|x_{i}\right|_{v}\right\} .
$$

2) Soit $\operatorname{Gal}\left(\mathbf{C}_{v} \mid k_{v}\right)$ l'ensemble des automorphismes continus de $\mathbf{C}_{v}$ qui laissent invariants les éléments de $k_{v}$. Alors $\|\cdot\|_{E, v}$ est invariante sous l'action de $\operatorname{Gal}\left(\mathbf{C}_{v} \mid k_{v}\right)$ : étant donné une $k_{v}$-base $\left(\alpha_{1}, \ldots, \alpha_{n}\right)$ de $E \otimes_{k} k_{v}$ et $\left(x_{1}, \ldots, x_{n}\right) \in$ $\mathbf{C}_{v}^{n}, \sigma \in \operatorname{Gal}\left(\mathbf{C}_{v} \mid k_{v}\right)$, on a

$$
\left\|\sigma\left(x_{1}\right) \alpha_{1}+\ldots+\sigma\left(x_{n}\right) \alpha_{n}\right\|_{\bar{E}, v}=\left\|x_{1} \alpha_{1}+\cdots+x_{n} \alpha_{n}\right\|_{\bar{E}, v} .
$$

3) Si $v$ est ultramétrique alors

$$
\forall x, y \in E \otimes_{k} \mathbf{C}_{v}, \quad\|x+y\|_{\bar{E}, v} \leq \max \left\{\|x\|_{\bar{E}, v},\|y\|_{\bar{E}, v}\right\} .
$$

Définition 2.2. Soit $\bar{E}$ un fibré vectoriel adélique sur Spec $k$ et $v$ une place de $k$. Nous dirons que $\bar{E}$ est $v$-pur si, pour tout $x \in E$, la norme $\|x\|_{\bar{E}, v}$ de $x$ appartient à l'image $\left|k_{v}\right|_{v}$ de $k_{v}$ par sa fonction valeur absolue. Lorsque cette propriété est satisfaite en toutes les places de $k$, nous dirons que $\bar{E}$ est pur.

Cette propriété est toujours vraie aux places archimédiennes de $k$ (lorsqu'il y en a). En vertu du premier article de la définition 2.1, vérifier que $\bar{E}$ est pur s'effectue sur un nombre fini de places ultramétriques de $k$. De plus, lorsqu'elle est satisfaite, elle l'est alors pour tout $x \in E \otimes_{k} k_{v}$ par densité topologique de $k$ dans $k_{v}$ et continuité des normes. L'étude des fibrés vectoriels adéliques purs et de leurs propriétés a été l'objet de l'article [6].

Définition 2.3. Un fibré adélique hermitien est un fibré vectoriel adélique pur dont toutes les normes aux places archimédiennes de $k$ sont hermitiennes.

Exemples. Pour tout $p \in[1,+\infty]$, on dispose du fibré vectoriel adélique pur $\left(k^{n},|\cdot|_{p}\right)$ (qui est hermitien lorsque $p=2$ ) où, pour toute place $v$ de $k$ et tout vecteur $\left(x_{1}, \ldots, x_{n}\right)$ de $\mathbf{C}_{v}^{n}$ on a

$$
\left|\left(x_{1}, \ldots, x_{n}\right)\right|_{p, v}:= \begin{cases}\left(\sum_{i=1}^{n}\left|x_{i}\right|_{v}^{p}\right)^{1 / p} & \text { si } v \text { est archimédienne et } p<+\infty \\ \max \left\{\left|x_{1}\right|_{v}, \ldots,\left|x_{n}\right|_{v}\right\} & \text { sinon. }\end{cases}
$$

Une autre famille d'exemples en lien avec la géométrie des nombres traditionnelle est présentée au $\S 6$. 
2.1.1. Défaut de pureté. Soit $\bar{E}$ un fibré vectoriel adélique $\operatorname{sur} \operatorname{Spec} k$, de dimension $n \geq 1$, et $v$ une place ultramétrique de $k$. Étant donné une $k_{v}$-base $\left(e_{1}, \ldots, e_{n}\right)$ de $E_{v}:=E \otimes_{k} k_{v}$, considérons le nombre réel $\delta_{0}\left(e_{1}, \ldots, e_{n}\right)$ défini comme la borne inférieure des produits $a b$ où $a$ et $b$ appartiennent à $\left|k_{v}\right|_{v}$ et satisfont à la condition suivante : pour tout $x=\sum_{i=1}^{n} x_{i} e_{i} \in E \otimes_{k} k_{v}$, on a

$$
a^{-1}\left|\left(x_{1}, \ldots, x_{n}\right)\right|_{\infty, v} \leq\|x\|_{\bar{E}, v} \leq b\left|\left(x_{1}, \ldots, x_{n}\right)\right|_{\infty, v} .
$$

De telles quantités $a, b$ existent toujours par équivalence des normes en dimension finie.

Définition 2.4. Le défaut de pureté de $\bar{E}$ en la place $v$ est la quantité $\delta\left(E_{v},\|\cdot\|_{\bar{E}, v}\right)$ définie comme la borne inférieure des $\delta_{0}\left(e_{1}, \ldots, e_{n}\right)$ lorsque $\left(e_{1}, \ldots, e_{n}\right)$ parcourt les $k_{v}$-bases de $E_{v}$.

Étant donné une uniformisante $\pi_{v}$ de l'anneau de valuation de $k_{v}$, l'image $\left|k_{v}\right|_{v}$ est le groupe multiplicatif monogène engendré par $\left|\pi_{v}\right|_{v}$. Comme $\delta_{0}\left(e_{1}, \ldots, e_{n}\right)$ et $\delta\left(E_{v},\|\cdot\|_{\bar{E}, v}\right)$ sont minorés (par 1), les bornes inférieures qui définissent ces nombres réels sont en réalité des minima. Ainsi $\delta_{0}\left(e_{1}, \ldots, e_{n}\right)$ et $\delta\left(E_{v},\|\cdot\|_{\bar{E}, v}\right)$ sont des puissances entières positives de $\left|\pi_{v}\right|_{v}^{-1}$.

Proposition 2.5. Avec les notations ci-dessus, on a $\delta\left(E_{v},\|\cdot\|_{\bar{E}, v}\right) \in\left\{1,\left|\pi_{v}\right|_{v}^{-1}\right\}$. De plus $\delta\left(E_{v},\|\cdot\|_{\bar{E}, v}\right)=1$ si et seulement si $\bar{E}$ est $v$-pur.

Démonstration. D'après la proposition 3 du chapitre II, p. 26, de [12], il existe une $k_{v}$-base $\left(\alpha_{1}, \ldots, \alpha_{n}\right)$ de $E \otimes_{k} k_{v}$ telle que, pour tout $\left(x_{1}, \ldots, x_{n}\right) \in k_{v}^{n}$, on a

$$
\left\|\sum_{j=1}^{n} x_{j} \alpha_{j}\right\|_{\bar{E}, v}=\max _{1 \leq j \leq n}\left\{\left|x_{j}\right|_{v}\left\|\alpha_{j}\right\|_{\bar{E}, v}\right\} .
$$

Pour chaque $i \in\{1, \ldots, n\}$, il existe un unique entier $n_{i}$ tel que $\left|\pi_{v}\right|_{v}^{n_{i}} \leq\left\|\alpha_{i}\right\|_{\bar{E}, v}<$ $\left|\pi_{v}\right|_{v}^{n_{i}-1}$. La base $\left(e_{1}, \ldots, e_{n}\right)$ constituée des vecteurs $e_{i}:=\alpha_{i} \pi_{v}^{-n_{i}}$ vérifie $\delta_{0}\left(e_{1}, \ldots, e_{n}\right) \leq$ $\left|\pi_{v}\right|_{v}^{-1}$. Cela implique que $\delta_{0}\left(e_{1}, \ldots, e_{n}\right)$ et $\delta\left(E_{v},\|\cdot\|_{\bar{E}, v}\right)$ appartiennent à $\left\{1,\left|\pi_{v}\right|_{v}^{-1}\right\}$. De plus si $\bar{E}$ est $v$-pur alors $\delta_{0}\left(e_{1}, \ldots, e_{n}\right)=1$ et donc $\delta\left(E_{v},\|\cdot\|_{\bar{E}, v}\right)=1$. Réciproquement, si $\delta\left(E_{v},\|\cdot\|_{\bar{E}, v}\right)=1$ alors il existe $a, b \in\left|k_{v}\right|_{v}$ qui vérifient (3) avec $a b=1$. Dans ce cas on a $\|x\|_{\bar{E}, v}=b\left|\left(x_{1}, \ldots, x_{n}\right)\right|_{\infty, v} \in\left|k_{v}\right|_{v}$ pour tout $x \in E_{v}$ et $\bar{E}$ est $v$-pur.

La définition du défaut de pureté a été calquée sur celle de la distance de BanachMazur entre deux espaces vectoriels normés de dimension finie (voir ci-dessous). Elle permet de comprendre immédiatement le fait suivant. Pour toute base $\left(e_{1}, \ldots, e_{n}\right)$ de $E_{v}$, il existe une matrice $c_{v} \in \mathrm{GL}_{n}\left(k_{v}\right)$ telle que, pour tout $x=\sum_{i=1}^{n} x_{i} e_{i} \in E_{v}$, on a

$$
\left|c_{v} X\right|_{\infty, v} \leq\|x\|_{\bar{E}, v} \leq \delta\left(E_{v},\|\cdot\|_{\bar{E}, v}\right)\left|c_{v} X\right|_{\infty, v} \quad \text { avec } X=\left(\begin{array}{c}
x_{1} \\
\vdots \\
x_{n}
\end{array}\right) .
$$

Définition 2.6. Le défaut de pureté de $\bar{E}$ est $\delta(\bar{E}):=\prod_{v} \delta\left(E_{v},\|\cdot\|_{\bar{E}, v}\right)^{n_{v}}$ (dans ce produit $v$ parcourt seulement les places ultramétriques de $k$ ).

Compte tenu des définitions, cette quantité $\delta(\bar{E})$ est plus grande que 1 et elle vaut 1 si et seulement si $\bar{E}$ est pur, en vertu de la proposition 2.5. 
2.1.2. Défaut d'hermitianité. Soit $\left(\mathrm{E}_{1},|\cdot| \mathrm{E}_{1}\right)$ et $\left(\mathrm{E}_{2},|\cdot| \mathrm{E}_{2}\right)$ deux espaces vectoriels réels (ou complexes) normés, de même dimension finie, et $T: \mathrm{E}_{1} \rightarrow \mathrm{E}_{2}$ une application linéaire, de norme d'opérateur

$$
\|T\|:=\sup \left\{\frac{|T(x)|_{\mathrm{E}_{2}}}{|x|_{E_{1}}} ; x \in \mathrm{E}_{1} \backslash\{0\}\right\} .
$$

La distance de Banach-Mazur entre $\left(\mathrm{E}_{1},|\cdot| \mathrm{E}_{1}\right)$ et $\left(\mathrm{E}_{2},|\cdot| \mathrm{E}_{2}\right)$, que l'on notera $\mathrm{d}\left(\left(\mathrm{E}_{1},|\cdot| \mathrm{E}_{1}\right),\left(\mathrm{E}_{2},|\cdot| \mathrm{E}_{2}\right)\right)$, est la borne inférieure des produits $\|T\| \cdot\left\|T^{-1}\right\|$ lorsque $T$ parcourt l'ensemble des isomorphismes entre $\mathrm{E}_{1}$ et $\mathrm{E}_{2}$.

Définition 2.7. Soit $\bar{E}$ un fibré vectoriel adélique sur $\operatorname{Spec} k$, de dimension $n \geq 1$. Le défaut d'hermitianité de $\bar{E}$, noté $\Delta(\bar{E})$, est le nombre réel

$$
\Delta(\bar{E}):=\prod_{v \text { archimédienne }} \mathrm{d}\left(\left(E \otimes_{k} k_{v},\|\cdot\|_{\bar{E}, v}\right),\left(k_{v}^{n},|\cdot|_{2, v}\right)\right)^{\left[k_{v}: \mathbf{R}\right]}
$$

si $k$ est un corps de nombres et $\Delta(\bar{E}):=1$ si $k$ est un corps de fonctions.

Un fibré adélique $\bar{E}$ est hermitien si et seulement si $\Delta(\bar{E})=\delta(\bar{E})=1$. Le théorème de John sur l'ellipsoïde de volume maximal dans un corps convexe permet de majorer $\Delta(\bar{E})$ (voir l'encadrement $(27)$ de [6]) :

$$
1 \leq \Delta(\bar{E}) \leq(2 n)^{D \delta / 2}
$$

2.1.3. Erratum à l'article [6]. Dans cet article et malheureusement sans que cela n'ait été mentionné, seuls des fibrés vectoriels adéliques purs interviennent. Plus précisément l'erreur se situe dans la formule (16) p. 39 de [6], qui est la formule (4) ici mais avec les normes des $\alpha_{i}$ toutes égales à 1 . Cette possibilité n'est offerte que si $\bar{E}$ est $v$-pur. Toutefois, l'erreur est facile à corriger. En effet, les démonstrations des énoncés de [6] ont presque toutes la même structure. On étudie au préalable le cas des fibrés adéliques hermitiens. Puis l'on passe au cas général. Il est possible de généraliser l'argument qui permet de s'affranchir simultanément des hypothèses de pureté et d'hermitianité de $\bar{E}$. Cela repose sur l'observation suivante : étant donné un fibré vectoriel adélique $\bar{E}$ sur Spec $k$ et un nombre réel $\varepsilon>0$, il existe une collection de normes $\left(\|\cdot\|_{\bar{E}, \varepsilon, v}\right)_{v}$ telles que

(i) $\bar{E}_{\varepsilon}:=\left(E,\left(\|\cdot\|_{\bar{E}, \varepsilon, v}\right)_{v}\right)$ est un fibré adélique hermitien $\operatorname{sur} \operatorname{Spec} k$,

(ii) pour toute place ultramétrique $v$ de $k$, pour tout $x \in E \otimes_{k} k_{v}$, on a

$$
\|x\|_{\bar{E}, \varepsilon, v} \leq\|x\|_{\bar{E}, v} \leq \delta\left(E_{v},\|\cdot\|_{\bar{E}, v}\right)\|x\|_{\bar{E}, \varepsilon, v},
$$

(iii) pour toute place archimédienne $v$ de $k$, pour tout $x \in E \otimes_{k} k_{v}$, on a

$$
\|x\|_{\bar{E}, \varepsilon, v} \leq\|x\|_{\bar{E}, v} \leq \mathrm{d}\left(\left(E_{v},\|\cdot\|_{\bar{E}, v}\right),\left(k_{v}^{n},|\cdot|_{2, v}\right)\right)(1+\varepsilon)\|x\|_{\bar{E}, \varepsilon, v} .
$$

L'existence de ces normes découle des définitions des défauts de pureté et d'hermitianité en une place $v$ (voir (5) lorsque $v$ est ultramétrique). De plus, comme $\delta\left(E_{v},\|\cdot\|_{\bar{E}, v}\right) \in\left|k_{v}\right|_{v}$, l'espace $E$ muni des normes qui sont à l'extrême droite des inégalités $(7)$ et (8) est encore un fibré adélique hermitien (et donc pur). Cela suffit pour que les énoncés de [6] se généralisent au cas non pur en remplaçant tout simplement $\Delta(\bar{E})$ par $\Delta(\bar{E}) \delta(\bar{E})$.

2.2. Hauteurs. Soit $\bar{E}$ un fibré vectoriel adélique $\operatorname{sur} \operatorname{Spec} k$. Soit $e=\left(e_{v}\right)_{v} \in$ $E \otimes_{k} k_{\mathbf{A}}$ et $r$ un adèle de $k$. La boule adélique de centre $e$ et de rayon $r$ est l'ensemble

$$
\mathbf{B}_{\bar{E}}(e, r):=\left\{x=\left(x_{v}\right)_{v} \in E \otimes_{k} k_{\mathbf{A}} ; \quad \forall v \text { place de } k,\left\|x_{v}-e_{v}\right\|_{\bar{E}, v} \leq\left|r_{v}\right|_{v}\right\} .
$$

En vertu de la première propriété des fibrés vectoriels adéliques, cette boule est compacte. Par abus de notation, on note 0 (resp. 1) l'adèle $(0, \ldots, 0, \ldots)$ (resp. l'idèle $(1, \ldots, 1, \ldots))$. Mesurer le volume de ce type d'ensemble requiert une mesure de Haar sur le groupe localement compact $\left(E \otimes_{k} k_{\mathbf{A}},+\right)$. Ces mesures ne différent 
que par un coefficient de proportionnalité strictement positif, et les définitions qui vont suivre sont faites de telle sorte qu'elles ne dépendent pas du choix de la mesure de Haar. Toutefois, pour des calculs précis, nous aurons besoin de la mesure produit $\mu=\prod_{v} \mu_{v}$ de mesures de Haar $\mu_{v}$ sur $\left(k_{v},+\right)$, normalisées de la manière suivante :

$\star$ si $v$ est réelle, $\mu_{v}$ est la mesure de Lebesgue $\mathrm{d} x$ normalisée sur $\mathbf{R}$,

* si $v$ est complexe et $\mathbf{C}$ identifié à $\mathbf{R}^{2}, \mu_{v}=2 \mathrm{~d} x \mathrm{~d} y$,

* si $v$ est ultramétrique et si $\mathcal{O}_{v}$ désigne l'anneau de valuation de $k_{v}$, i.e. $\mathcal{O}_{v}=\left\{x \in k_{v} ;|x|_{v} \leq 1\right\}$, alors $\mu_{v}\left(\mathcal{O}_{v}\right)=1$.

La mesure de Haar induite par $\mu$ sur le quotient compact $k_{\mathbf{A}} / k$ attribue à celui-ci une mesure égale à $\left|D_{k}\right|^{1 / 2}$ si $k$ est un corps de nombres de discriminant absolu $D_{k}$ et une mesure égale à $q^{g(k)-1}$ si $k$ est un corps de fonctions de genre $g(k)$ et si $q$ désigne le cardinal du plus grand corps fini inclus dans $k$ (voir chapitres 5 et 6 de [12]). La mesure $\mu$ sur $k_{\mathbf{A}}$ confère à $E \otimes_{k} k_{\mathbf{A}}$ une mesure de Haar $\mu_{E}$ via le choix d'une $k$-base de $E$ (dont la mesure $\mu_{E}$ ne dépend pas in fine, grâce à la formule du produit).

Définition 2.8. Soit vol une mesure de Haar sur $\left(k_{\mathbf{A}}^{n},+\right)$ et $\phi: E \rightarrow k^{n}$ un isomorphisme de $k$-espaces vectoriels. La hauteur de $\bar{E}$ est le nombre réel

$$
H(\bar{E}):=\frac{\operatorname{vol}\left(\mathbf{B}_{\left(k^{n},||_{2}\right)}(0,1)\right)}{\operatorname{vol}\left(\phi\left(\mathbf{B}_{\bar{E}}(0,1)\right)\right)}
$$

(si $E=\{0\}$ alors $H(\bar{E}):=1$ ).

Cette définition ne dépend pas des choix de la mesure de Haar et de l'isomorphisme $\phi$ (formule du produit). Elle est compatible avec celle donnée dans l'introduction lorsque $\bar{E}$ est hermitien (voir [6], corollaire 4.10). Soit $r_{1}, r_{2}$ le nombre de places réelles et complexes respectivement de $k$ ( si $\delta=0$ alors $\left.r_{1}=r_{2}=0\right)$. Soit $V_{n}$ le volume de la boule euclidienne de rayon 1 pour la mesure de Lebesgue usuelle sur $\mathbf{R}^{n}$, i.e.

$$
V_{n}=\frac{\pi^{n / 2}}{\Gamma(1+n / 2)}
$$

Alors, au moyen de la mesure $\mu_{E}$ et de la définition 2.8, se prouve l'égalité

$$
\frac{\operatorname{covol}(E)}{\operatorname{vol}\left(\mathbf{B}_{\bar{E}}(0,1)\right)}=\frac{H(\bar{E})}{2^{n r_{2}} V_{n}^{r_{1}} V_{2 n}^{r_{2}}} \times \begin{cases}\left|D_{k}\right|^{n / 2} & \text { si } \delta=1, \\ q^{n(g(k)-1)} & \text { si } \delta=0,\end{cases}
$$

pour tout fibré vectoriel adélique $\bar{E}$ sur Spec $k$ de dimension $n \geq 1$ et toute mesure de Haar vol sur $E \otimes_{k} k_{\mathbf{A}}$. Dans cet énoncé, $\operatorname{covol}(E)$ désigne la mesure de Haar du quotient $\left(E \otimes_{k} k_{\mathbf{A}}\right) / E$ induite par la mesure de Haar vol sur $E \otimes_{k} k_{\mathbf{A}}$.

Définition 2.9. Soit $x \in E$. La hauteur de $x$, relative $\grave{a} \bar{E}$, est $H_{\bar{E}}(x):=\prod_{v}\|x\|_{\bar{E}, v}^{n_{v}}$ $\left(n_{v}\right.$ est le degré local).

Les définitions 2.8 et 2.9 se relient entre elles par la formule $H_{\bar{E}}(x)=H\left(k \cdot x,\left(\|\cdot\|_{\bar{E}, v}\right)_{v}\right)$.

2.3. Inégalité de van der Corput adélique. Soit $\bar{E}$ un fibré vectoriel adélique sur Spec $k$. La discrétude de $E$ dans $E \otimes_{k} k_{\mathbf{A}}$ et la compacité de la boule adélique $\mathbf{B}_{\bar{E}}(0, r)$ font de l'intersection $E \cap \mathbf{B}_{\bar{E}}(0, r)$ un ensemble fini pour tout $r \in k_{\mathbf{A}}$. Ce paragraphe vise à établir une minoration simple du cardinal de $E \cap \mathbf{B}_{\bar{E}}(0, r)$, pour $r \in k_{\mathbf{A}}^{\times}$, en fonction de la hauteur de $\bar{E}$. Commençons par une version adélique d'un théorème classique de Blichfeldt.

Proposition 2.10. Soit $k$ un corps global et $\ell \in \mathbf{N} \backslash\{0\}$. Soit $\bar{E}$ un fibré vectoriel adélique sur $\operatorname{Spec} k$, de dimension $n \geq 1$. Soit $e \in E \otimes k_{\mathbf{A}}$. Supposons que

$$
\ell \operatorname{covol}(E)<\operatorname{vol}\left(\mathbf{B}_{\bar{E}}(e, 1)\right) \text {. }
$$


Alors il existe $\ell+1$ points distincts $e_{0}, \ldots, e_{\ell}$ de $\mathbf{B}_{\bar{E}}(e, 1)$ tels que, pour tous $i, j \in$ $\{0, \ldots, \ell\}$, on $a e_{i}-e_{j} \in E$. Si $k$ est un corps de nombres, cet énoncé reste vrai lorsqu'il y a égalité dans (10).

Démonstration. Posons $C:=\mathbf{B}_{\bar{E}}(e, 1)$ et $E_{\mathbf{A}}:=E \otimes_{k} k_{\mathbf{A}}$. Soit $f: E_{\mathbf{A}} \rightarrow\{0,1\}$ la fonction caractéristique de $C$. Soit $P$ un domaine fondamental de $E_{\mathbf{A}} / E$ (voir le chapitre $\mathrm{V}$ de [12]). Pour $x \in E_{\mathbf{A}}$, il n'y a qu'un nombre fini d'éléments $\lambda \in$ $E \cap(C-x)$. Par conséquent, la somme $\sum_{\lambda \in E} f(x+\lambda)$ est finie et l'on peut définir une fonction (mesurable) $F: P \rightarrow \mathbf{N}$ qui à $x \in P$ associe cette somme. On a

$$
\begin{aligned}
\int_{P} F(x) \mathrm{d} x & =\sum_{\lambda \in E} \int_{P} f(x+\lambda) \mathrm{d} x \\
& =\sum_{\lambda \in E} \int_{P+\lambda} f(x) \mathrm{d} x \\
& =\int_{\bigcup_{\lambda \in E}(P+\lambda)=E_{\mathbf{A}}} f(x) \mathrm{d} x=\operatorname{vol}(C) .
\end{aligned}
$$

Lorsque $\operatorname{vol}(C)>\ell \operatorname{covol}(E)$, on a $\int_{P}(F(x)-\ell) \mathrm{d} x>0$ et il existe $x \in P$ tel que $F(x)>\ell$, i.e. $F(x) \geq \ell+1$. Dans ce cas, il existe des vecteurs $\lambda_{0}, \ldots, \lambda_{\ell} \in E$, distincts, tels que $e_{i}:=x+\lambda_{i} \in C$ et la proposition est démontrée. Si $k$ est un corps de nombres et $\operatorname{si} \operatorname{vol}(C)=\ell \operatorname{covol}(E)$, on choisit une place archimédienne $v_{0}$ de $k$ et, au moyen d'un $\varepsilon \in] 0,1$ [, l'on forme le fibré vectoriel adélique $\overline{E_{\varepsilon}}$ identique à $\bar{E}$ sauf pour la norme $\|\cdot\|_{\bar{E}, v_{0}}$ qui est remplacée par $(1-\varepsilon)\left\|_{\cdot}\right\|_{\bar{E}, v_{0}}$. On a alors

$$
\operatorname{vol}\left(\mathbf{B}_{\overline{E_{\varepsilon}}}(e, 1)\right)=\left(\frac{1}{1-\varepsilon}\right)^{n n_{v_{0}}} \operatorname{vol}(C)>\ell \operatorname{covol}(E) .
$$

D'après le cas déjà traité, il existe $e_{0}^{(\varepsilon)}, \ldots, e_{\ell}^{(\varepsilon)} \in \mathbf{B}_{\overline{E_{\varepsilon}}}(e, 1)$, distincts, tels que $e_{i}^{(\varepsilon)}-e_{j}^{(\varepsilon)} \in E$. Soit $2 v_{0}$ l'idèle composé de 2 à la place $v_{0}$ et de 1 aux autres places de $k$. Pour $\varepsilon<1 / 2$, tous les $e_{i}^{(\varepsilon)}, i \in\{0, \ldots, \ell\}$, appartiennent à la boule compacte $\mathbf{B}_{\bar{E}}\left(e, 2_{v_{0}}\right)$. Il existe donc une suite $\varepsilon_{h} \rightarrow 0$ et $e_{0}, \ldots, e_{\ell} \in E_{\mathbf{A}}$ tels que $e_{i}^{\left(\varepsilon_{h}\right)} \rightarrow e_{i}$ lorsque $h \rightarrow+\infty$. On a clairement $e_{i} \in \mathbf{B}_{\bar{E}}(e, 1)$. De plus, comme tous les $e_{i}^{(\varepsilon)}-e_{j}^{(\varepsilon)}$, pour $i \neq j$, sont des éléments de $E$ de hauteur bornée, les valeurs prises par ces différences sont toutes non nulles et en nombre fini. Par conséquent, pour tous $i \neq j \in\{1, \ldots, \ell\}$, le vecteur $e_{i}-e_{j}$ est égal à une de ces valeurs et $e_{i}-e_{j} \in E \backslash\{0\}$.

On en déduit alors une variante du théorème 3 de [10].

Corollaire 2.11. Soit $\ell \in \mathbf{N}$ et $\bar{E}$ un fibré vectoriel adélique sur Spec $k$. Soit $r \in k_{\mathbf{A}}^{\times}$. Supposons que l'une des deux hypothèses suivantes est satisfaite :

(i) $2^{n D} \ell \operatorname{covol}(E) \leq \operatorname{vol}\left(\mathbf{B}_{\bar{E}}(0, r)\right)$ si $k$ est un corps de nombres,

(ii) $\ell \operatorname{covol}(E)<\operatorname{vol}\left(\mathbf{B}_{\bar{E}}(0, r)\right)$ si $k$ est un corps de fonctions.

Alors on $a \ell+1 \leq \operatorname{card}\left(E \cap \mathbf{B}_{\bar{E}}(0, r)\right)$.

Dans le cas classique de l'intersection d'un réseau et d'une boule euclidienne, on dispose d'une minoration un peu plus forte avec $2 \ell+1$ au lieu de $\ell+1$, connue alors comme l'inégalité de van der Corput.

Démonstration. Soit $r=\left(r_{v}\right)_{v} \in k_{\mathbf{A}}^{\times}$. Soit $\bar{F}$ le fibré vectoriel adélique obtenu à partir de $\bar{E}$ en multipliant par $2 /\left|r_{v}\right|_{v}$ les normes de $\bar{E}$ aux places archimédiennes de $k$ et par $1 /\left|r_{v}\right|_{v}$ les normes de $\bar{E}$ en les autres places. On a $\operatorname{vol}\left(\mathbf{B}_{\bar{F}}(0,1)\right)=$ $\operatorname{vol}\left(\mathbf{B}_{\bar{E}}(0, r)\right) / 2^{n D \delta}$. On conclut en appliquant la proposition 2.10 avec $e=0$. 
Ce corollaire avec $\ell=1$ correspond au premier théorème de Minkowski, qui peut être vu comme un lemme de Siegel simple, sans condition. En choisissant pour $\ell$ la partie entière de $\operatorname{vol}\left(\mathbf{B}_{\bar{E}}(0, r)\right) /\left(2^{n D \delta} \operatorname{covol}(E)\right)$, éventuellement diminuée de 1 si ce quotient est entier et si $\delta=0$, on obtient le

Corollaire 2.12. Soit $\bar{E}$ un fibré vectoriel adélique sur $\operatorname{Spec} k$, de dimension $n \geq 1$, et $r \in k_{\mathbf{A}}^{\times}$. Alors on a

$$
\frac{\operatorname{vol}\left(\mathbf{B}_{\bar{E}}(0, r)\right)}{2^{n D \delta} \operatorname{covol}(E)} \leq \operatorname{card}\left(E \cap \mathbf{B}_{\bar{E}}(0, r)\right) .
$$

2.4. Minima successifs. Soit $\bar{E}$ un fibré vectoriel adélique sur Spec $k$, de dimension $n \geq 1$. Si $r=\left(r_{v}\right)_{v} \in k_{\mathbf{A}}$, on note $|r|_{\mathbf{A}}$ le produit (parfois nul) $\prod_{v}\left|r_{v}\right|_{v}^{n_{v}}$, appelé module de $r$.

Pour tout $x \in E \backslash\{0\}$, on a $\|x\|_{\bar{E}, v}=1$ en dehors d'un nombre fini de places, en vertu de la première propriété de la définition 2.1. Par conséquent, si e := $\left(e_{1}, \ldots, e_{n}\right)$ est une $k$-base de $E$, il existe un idèle $r=\left(r_{v}\right)_{v}$ tel que $\left|r_{v}\right|_{v} \geq$ $\max _{1 \leq i \leq n}\left\{\left\|e_{i}\right\|_{\bar{E}, v}\right\}$, pour toute place $v$. La boule adélique $\mathbf{B}_{\bar{E}}(0, r)$ contient alors tous les vecteurs de la base $\mathbf{e}$. Cette observation légitime la définition suivante.

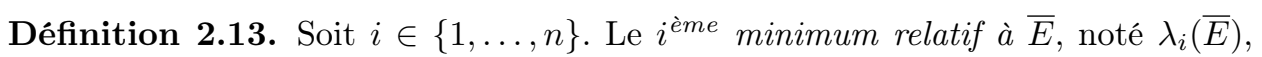
est la borne inférieure de l'ensemble des nombres réels positifs de la forme $|r|_{\mathbf{A}}$ où $r \in k_{\mathbf{A}}$ est tel que $E \cap \mathbf{B}_{\bar{E}}(0, r)$ contienne $i$ vecteurs $k$-linéairement indépendants.

Empruntée à Thunder [10], cette définition est légèrement différente de celle de Bombieri \& Vaaler [2] qui n'autorisent que le cas d'un corps de nombres avec des idèles $r$ tels que $r_{v}=1$ aux places ultramétriques et $r_{v}$ constant aux places archimédiennes, égal à un nombre réel $>0$ fixé. Un tel idèle sera dit de BombieriVaaler et nous noterons $\lambda_{i}^{\mathrm{BV}}(\bar{E})$ la puissance $[k: \mathbf{Q}]$-ème du $i^{\text {ème }}$ minimum de ces auteurs. Mais la définition 2.13 est a priori encore plus éloignée de celle que l'on trouve par exemple dans [9] et [11], où est défini le nombre réel $\Lambda_{i}(\bar{E})$ comme la borne inférieure des nombres réels $\lambda$ pour lesquels il existe une famille libre de $E$ à $i$ éléments et dont chaque élément est de hauteur plus petite que $\lambda$. Comme il n'y a qu'un nombre fini de valeurs prises par la fonction hauteur à l'intérieur d'un compact de $\mathbf{R}$ (Northcott), il existe une $k$-base $\left(e_{1}, \ldots, e_{n}\right)$ de $E$ telle que, pour tout $i \in\{1, \ldots, n\}, \Lambda_{i}(\bar{E})=H_{\bar{E}}\left(e_{i}\right)$. Cette propriété montre que $\Lambda_{i}(\bar{E})$ et $\lambda_{i}(\bar{E})$ sont en général distincts. Par exemple, si $k$ est un corps de fonctions, chaque module $|r|_{\mathbf{A}}$ est une puissance entière de la caractéristique de $k$. Il en est alors de même pour $\lambda_{i}(\bar{E})$ qui est alors un minimum. Mais la possibilité de choisir des normes impures quelconques (en multipliant par exemple la norme du max sur les coordonnées dans une base de $E$ par un nombre transcendant) en un nombre fini de places de $k$ montre que $\Lambda_{i}(\bar{E})$ n'est pas en général une puissance de $p$. On a les inégalités

$$
\forall i \in\{1, \ldots, n\}, \quad \Lambda_{i}(\bar{E}) \leq \lambda_{i}(\bar{E}) \leq \lambda_{i}^{\mathrm{BV}}(\bar{E})
$$

(cette dernière quantité n'existe que si $k$ est un corps de nombres contrairement aux deux autres minima).

La notion de pureté se révèle utile dans les énoncés suivants.

Proposition 2.14. Soit $k$ un corps global et $\bar{E}$ un fibré vectoriel adélique sur Spec $k$. Si $\bar{E}$ est pur alors $\lambda_{1}(\bar{E})=\Lambda_{1}(\bar{E})$.

Démonstration. Soit $e_{1} \in E$ tel que $H_{\bar{E}}\left(e_{1}\right)=\Lambda_{1}(\bar{E})$. Comme $\bar{E}$ est pur, il existe un idèle $r=\left(r_{v}\right) \in k_{\mathbf{A}}^{\times}$tel que $\left|r_{v}\right|_{v}=\left\|e_{1}\right\|_{\bar{E}, v}$ pour toute place $v$ de $k$. Le vecteur $e_{1}$ appartient à la boule adélique $\mathbf{B}_{\bar{E}}(0, r)$ et donc $\lambda_{1}(\bar{E}) \leq|r|_{\mathbf{A}}=\Lambda_{1}(\bar{E})$. 
Cette proposition ne s'étend pas en général aux autres minima, comme le prouve l'exemple suivant, que m'a suggéré Gaël Rémond.

Exemple 2.15. Soit $k=\mathbf{Q}(\sqrt{2})$ et $w, w^{\prime}$ ses deux places archimédiennes : $\mid a+$ $\left.b \sqrt{2}\right|_{w}=|a+b \sqrt{2}|$ et $|a+b \sqrt{2}|_{w^{\prime}}=|a-b \sqrt{2}|$. Posons $r_{0}:=(1+\sqrt{2}) / 2$. Soit $\bar{E}$ le fibré vectoriel adélique sur $k$ d'espace vectoriel $k^{2}$ et de normes :

$$
\forall(x, y) \in k_{v}^{2}, \quad\|(x, y)\|_{\bar{E}, v}:= \begin{cases}\max \left\{|x|_{v},|y|_{v} / 2\right\} & \text { si } v=w, \\ \max \left\{|x|_{v}, 2|y|_{v}\right\} & \text { si } v=w^{\prime}, \\ \max \left\{|x|_{v},|y|_{v}\right\} & \text { si } v \text { est ultramétrique. }\end{cases}
$$

Alors on a $\Lambda_{1}(\bar{E})=\lambda_{1}(\bar{E})=\lambda_{1}^{\mathrm{BV}}(\bar{E})=1$ tandis que $\Lambda_{2}(\bar{E})=1, \lambda_{2}(\bar{E})=r_{0}$ et $\lambda_{2}^{\mathrm{BV}}(\bar{E})=r_{0}^{2}$.

Démonstration. Tout d'abord, la formule du produit implique la minoration $1 \leq$ $H_{\bar{E}}(x, y)$ pour tout $(x, y) \in E \backslash\{0\}$. Comme il y a égalité pour les vecteurs $(1,0)$ et $(0,1)$, on a l'égalité des premiers minima et de $\Lambda_{2}(\bar{E})$ avec 1 . De plus les vecteurs $(1,0)$ et $(0,1+\sqrt{2})$ forment une base de $E$. Ils appartiennent aux boules adéliques de rayons $r=\left(r_{0}, 1,1, \ldots\right)$ et $r^{\prime}=\left(r_{0}, r_{0}, 1, \ldots\right)$. Ceci montre que $\lambda_{2}(\bar{E})$ et $\lambda_{2}^{\mathrm{BV}}(\bar{E})$ sont plus petits que $r_{0}$ et $r_{0}^{2}$ respectivement. Par ailleurs, soit $r=\left(r_{v}\right)_{v} \in k_{\mathbf{A}}$ tel que la boule adélique $\mathbf{B}_{\bar{E}}(0, r)$ contienne une base de $E$. En observant que, pour toute place $v$ de $k$ et pour tout $(x, y) \in E \backslash\{0\}$, on a

$$
\max \left\{\|(x, 0)\|_{\bar{E}, v},\|(0, y)\|_{\bar{E}, v}\right\}=\|(x, y)\|_{\bar{E}, v},
$$

on peut supposer que cette base est de la forme $\{(x, 0),(0, y)\}$ avec $x y \neq 0$. Quitte à remplacer $r$ par $r / x$, en conservant le même module, on peut supposer que $x=1$. Ces considérations montrent que $\lambda_{2}(\bar{E})$ est la borne inférieure pour $(a, b) \in \mathbf{Q}^{2} \backslash\{0\}$ des produits

$$
\lambda_{2}(a, b):=\max \{1,|a+b \sqrt{2}| / 2\} \max \{1,2|a-b \sqrt{2}|\} \prod_{v \nmid \infty} \max \left\{1,|a+b \sqrt{2}|_{v}\right\}^{n_{v}}
$$

et que $\lambda_{2}^{\mathrm{BV}}(\bar{E})$ est la borne inférieure sur la même expression mais où le produit des deux premiers maxima est remplacé par $\max \{1,|a+b \sqrt{2}| / 2,2|a-b \sqrt{2}|\}^{2}$. Ces bornes inférieures sont des minima par propriété de hauteur. Étant donné un nombre premier $p$ et une place $v$ de $k$ au-dessus de $p$, si $|a+b \sqrt{2}|_{v}>1$ alors $|a+b \sqrt{2}|_{v}^{n_{v}} \geq p^{n_{v} / 2} \geq 2$. Cette dernière minoration est évidente si $p \geq 5$. Mais si $p \in\{2,3\}$ alors on vérifie que le degré local $n_{v}$ vaut 2 (en montrant par exemple que $X^{2}-2$ n'a pas de racine dans $\mathbf{Q}_{p}$ ) et l'on a encore $p^{n_{v} / 2} \geq 2$. Dans tous les cas, si $a+b \sqrt{2}$ n'est pas dans l'anneau de valuation de $k_{v}$ alors $\lambda_{2}(a, b) \geq 2$. Donc si $\lambda_{2}(a, b)=\lambda_{2}(\bar{E})$ (ce que l'on suppose dorénavant), que l'on sait inférieur à $r_{0}<2$, l'élément $a+b \sqrt{2}$ est un entier de $k$, i.e. il appartient à $\mathbf{Z}[\sqrt{2}]$. Le produit des deux premiers maxima dans $\lambda_{2}(a, b)$ est supérieur à $\left|a^{2}-2 b^{2}\right|$, qui est un entier strictement positif et plus petit que $r_{0}$. Il vaut donc 1 , ce qui signifie que $a+b \sqrt{2}$ est une unité de $\mathbf{Z}[\sqrt{2}]$. Le groupe des unités de cet anneau est monogène engendré par $1+\sqrt{2}$. Ainsi il existe $n \in \mathbf{Z}$ tel que $a+b \sqrt{2}=(1+\sqrt{2})^{n}$. Comme $\lambda_{2}(\bar{E}) \leq r_{0}$ on a $|a+b \sqrt{2}| \leq 2 r_{0}$ donc $n \leq 1$. De la même manière on a $(1+\sqrt{2})^{-n}=|a-b \sqrt{2}| \leq r_{0} / 2$ donc $-n<0$ et finalement $n=1$, puis $\lambda_{2}(\bar{E})=\lambda_{2}(1,1)=r_{0}$. Le calcul de $\lambda_{2}^{\overline{\mathrm{BV}}}(\bar{E})$ repose sur les mêmes arguments, car $r_{0}^{2}$ est encore strictement plus petit que 2 .

Bien que distincts en général, ces différents minima restent toutefois comparables, comme l'ont déjà remarqué Christensen \& Gubler (voir lemme 2.11 de [3]) :

Proposition 2.16. Soit $\bar{E}$ un fibré vectoriel adélique pur sur $\operatorname{Spec} k$, de dimension $n \geq 1$. Si $k$ est un corps de nombres, avec $r_{2}$ places complexes non réelles, alors, pour tout $i \in\{1, \ldots, n\}$, on a $\lambda_{i}^{\mathrm{BV}}(\bar{E}) \leq\left(\frac{2}{\pi}\right)^{r_{2}}\left|D_{k}\right|^{1 / 2} \Lambda_{i}(\bar{E})$. Si $k$ est un corps de 
fonctions de genre $g(k)$ et si $q$ désigne le cardinal du plus grand corps fini inclus dans $k$, alors, pour tout $i \in\{1, \ldots, n\}$, on a $\lambda_{i}(\bar{E}) \leq q^{g(k)} \Lambda_{i}(\bar{E})$. Dans le cas général d'un fibré vectoriel adélique $\bar{E}$ qui n'est pas nécessairement pur, ces résultats restent valides à condition de multiplier les majorants par $\delta(\bar{E})$.

Démonstration. Supposons dans un premier temps que $\bar{E}$ est pur. Soit $\left\{e_{1}, \ldots, e_{n}\right\}$ une base de $E$ telle que, pour tout $j \in\{1, \ldots, n\}$, on a $H_{\bar{E}}\left(e_{j}\right)=\Lambda_{j}(\bar{E})$. Soit $r_{j}=$ $\left(r_{j, v}\right)_{v}$ un idèle tel que $\left|r_{j, v}\right|_{v}=\left\|e_{j}\right\|_{\bar{E}, v}$ pour toute place $v$ de $k$. Soit $i \in\{1, \ldots, n\}$. Si $k$ est un corps de nombres, on pose

$$
\lambda:=\left(\left(\frac{2}{\pi}\right)^{r_{2}}\left|D_{k}\right|^{1 / 2} \Lambda_{i}(\bar{E})\right)^{1 / D}
$$

et $r=(\lambda, \ldots, \lambda, 1,1, \ldots)$ l'idèle de $k$ avec pour composantes $\lambda$ aux places archimédiennes de $k$ et 1 aux autres places. Si $k$ est un corps de fonctions, soit $r \in k_{\mathbf{A}}$ tel que $|r|_{\mathbf{A}}=q^{g(k)} \Lambda_{i}(\bar{E})$. Un tel choix est possible car $\bar{E}$ est pur et car $\left|k_{\mathbf{A}}^{\times}\right|_{\mathbf{A}}$ est l'ensemble $q^{\mathbf{Z}}$. Pour toute mesure de Haar vol sur $k_{\mathbf{A}}$, la formule (9) du $\S 2.2$ appliquée à $\left(k,\left(|\cdot|_{v}\right)_{v}\right)$ donne

$$
\frac{\operatorname{covol}(k)}{\operatorname{vol}\left(\mathbf{B}_{\left(k,\left(|\cdot|_{v}\right)_{v}\right)}(0,1)\right)}= \begin{cases}\frac{\left|D_{k}\right|^{1 / 2}}{2^{D-r_{2} \pi^{r} 2}} & \text { si } k \text { est un corps de nombres } \\ q^{g(k)-1} & \text { si } k \text { est un corps de fonctions. }\end{cases}
$$

Ainsi, en vertu du corollaire 2.11 appliqué à $\left(k,\left(|\cdot|_{v}\right)_{v}\right)$ et avec le rayon $r / r_{j}$, il existe $\eta_{j} \in k \backslash\{0\}$ tel que, pour toute place $v$ de $k$, on a $\left\|\eta_{j} e_{j}\right\|_{\bar{E}, v} \leq\left|r_{v}\right|_{v}$. La famille libre $\left\{\eta_{1} e_{1}, \ldots, \eta_{i} e_{i}\right\}$ est donc incluse dans la boule $\mathbf{B}_{\bar{E}}(0, r)$ et la proposition s'ensuit. Lorsque $\bar{E}$ n'est pas pur, l'argumentation du $\S 2.1 .3$ montre qu'il existe un fibré adélique pur $\overline{\mathrm{E}}:=\left(E,\left(\|\cdot\|_{\overline{\mathrm{E}}_{,}}\right)_{v}\right)$ tel que $\|\cdot\|_{\overline{\mathrm{E}}_{,}}=\|\cdot\|_{\bar{E}, v}$ si $v$ est archimédienne et $\|\cdot\|_{\overline{\mathrm{E}}, v} \leq\|\cdot\|_{\bar{E}, v} \leq \delta\left(E_{v},\|\cdot\|_{\bar{E}, v}\right)\|\cdot\|_{\overline{\mathrm{E}}, v}$ sinon. Il suffit alors d'observer que, pour tout $i \in\{1, \ldots, n\}$, si $\lambda$ désigne une des trois fonctions $\Lambda_{i}, \lambda_{i}, \lambda_{i}^{\mathrm{BV}}$, on a $\lambda(\overline{\mathrm{E}}) \leq \lambda(\bar{E}) \leq$ $\delta(\bar{E}) \lambda(\overline{\mathrm{E}})$ pour conclure, en se ramenant au cas pur.

Corollaire 2.17. Soit $\bar{E}$ un fibré vectoriel adélique pur sur $\operatorname{Spec} k$. On suppose que $k=\mathbf{Q}$ ou que $k=k_{0}(T)$ avec $k_{0}$ un corps fini. Alors, pour tout entier $i \in\{1, \ldots, n\}$, on $a \Lambda_{i}(\bar{E})=\lambda_{i}(\bar{E})=\lambda_{i}^{\mathrm{BV}}(\bar{E})$.

Démonstration. Les hypothèses faites sur $k$ assurent que $r_{2}=0$ et $D_{k}=1$ si $k$ est un corps de nombres, et $g(k)=0$ dans l'autre cas. Le corollaire découle alors de la proposition 2.16 et de la comparaison (11).

S'il existe une variante du second théorème de Minkowski, due à E. Bombieri \& J. Vaaler [2], qui majore le produit $\lambda_{1}(\bar{E}) \cdots \lambda_{n}(\bar{E})$ (voir inégalité (15)), nous aurons surtout besoin d'une minoration, beaucoup plus élémentaire à obtenir. Il s'agit de la proposition 4.13 de [6], généralisée au cas impur comme nous l'avons expliqué au $\S 2.1 .3$.

Proposition 2.18. Soit $\bar{E}$ un fibré vectoriel adélique sur $\operatorname{Spec} k$, de dimension $n \geq 1$. On $a$

$$
\frac{H(\bar{E})}{(\Delta(\bar{E}) \delta(\bar{E}))^{n}} \leq \lambda_{1}(\bar{E}) \cdots \lambda_{n}(\bar{E})
$$

(le membre de droite peut être remplacé par le produit $\Lambda_{1}(\bar{E}) \cdots \Lambda_{n}(\bar{E})$ ).

2.5. Version adélique d'un théorème de Henk. Dans tout ce paragraphe, $k$ est un corps de nombres de degré $D$. 
Théorème 2.19. Soit $\bar{E}$ un fibré vectoriel adélique sur $\operatorname{Spec} k$ et $\bar{F} \subseteq \bar{E}$ un sousfibré adélique de dimension $m \geq 1$. Soit $\lambda_{1}(\bar{F}) \leq \cdots \leq \lambda_{m}(\bar{F})$ les minima successifs de $\bar{F}$. Alors, pour tout $r \in k_{\mathbf{A}}$, on a

$$
\operatorname{card}\left(F \cap \mathbf{B}_{\bar{E}}(0, r)\right)<2^{3 m D-m-1} \prod_{i=1}^{m}\left(1+\frac{|r|_{\mathbf{A}}}{\lambda_{i}(\bar{F})}\right) .
$$

Lorsque $r$ est un idèle de Bombieri-Vaaler $\left(^{*}\right)$, on peut remplacer $\lambda_{i}(\bar{F})$ par $\lambda_{i}^{\mathrm{BV}}(\bar{F})$ dans ce majorant.

La démonstration consiste à se ramener au cas d'un réseau de $\mathbf{R}^{n D}$ intersecté avec un corps convexe symétrique, cadre dans lequel se situe l'énoncé original [7] de M. Henk.

Démonstration du théorème 2.19. On peut supposer que $\bar{F}=\bar{E} \operatorname{car} \operatorname{card}(F \cap$ $\left.\mathbf{B}_{\bar{E}}(0, r)\right)=\operatorname{card}\left(F \cap \mathbf{B}_{\bar{F}}(0, r)\right)$. On note $\infty$ la place archimédienne de $\mathbf{Q}$. Soit $r=\left(r_{v}\right)_{v} \in k_{\mathbf{A}}$. On peut supposer que $r \in k_{\mathbf{A}}^{\times}$car, dans le cas contraire, l'intersection $F \cap \mathbf{B}_{\bar{E}}(0, r)$ est réduite à $\{0\}$ et le théorème est évident. Considérons l'ensemble

$$
C:=\prod_{v \mid \infty}\left\{x \in E \otimes_{k} k_{v} ;\|x\|_{\bar{E}, v} \leq\left|r_{v}\right|_{v}\right\} .
$$

Ce sous-ensemble de $\mathfrak{E}:=\prod_{v \mid \infty} E \otimes_{k} k_{v}$ est convexe, compact, d'intérieur non vide et symétrique par rapport à l'origine. Via le plongement diagonal

$$
\iota: E \hookrightarrow E \otimes_{\mathbf{Q}} \mathbf{R} \stackrel{\sim}{\longrightarrow} \mathfrak{E},
$$

l'ensemble $\mathcal{O}:=\left\{x \in E ; \forall v \nmid \infty,\|x\|_{\bar{E}, v} \leq\left|r_{v}\right|_{v}\right\}$ est un réseau (complet) de $\mathfrak{E}$ car, pour tout $x \in E$, il existe un entier $N>0$ tel que $N x \in \mathcal{O}$. Les ensembles $E \cap \mathbf{B}_{\bar{E}}(0, r)$ et $C \cap \iota(\mathcal{O})$ sont en bijection et il s'agit alors de majorer $\operatorname{card}(C \cap \iota(\mathcal{O}))$. Dans ces conditions, on peut définir, pour tout $i \in\{1, \ldots, n D\}$, le nombre réel $\lambda_{i}(C, \mathcal{O})$ comme la borne inférieure des nombres réels $\lambda>0$ tels que $\lambda C \cap \iota(\mathcal{O})$ possède $i$ vecteurs $\mathbf{R}$-linéairement indépendants. Ce nombre est un minimum car les intersections $\lambda C \cap \iota(\mathcal{O})$ sont finies et contenues les unes dans les autres lorsque $\lambda$ décroît. Pour tout $\ell \in\{0,1, \ldots, n-1\}$, on a

$$
\lambda_{\ell+1}(\bar{E}) \leq \lambda_{\ell D+1}(C, \mathcal{O})^{D}|r|_{\mathbf{A}} .
$$

En effet, considérons $\ell D+1$ vecteurs $\iota\left(u_{1}\right), \ldots, \iota\left(u_{\ell D+1}\right)$ de $\left(\lambda_{\ell D+1}(C, \mathcal{O}) C\right) \cap$ $\iota(\mathcal{O})$ linéairement indépendants sur $\mathbf{R}$. Par construction, les éléments $u_{i}$ appartiennent à $E \cap \mathbf{B}_{\bar{E}}\left(0, r \lambda_{\mathbf{A}}\right)$ où $\lambda_{\mathbf{A}}$ désigne l'idèle de Bombieri-Vaaler de composante $\lambda_{\ell D+1}(C, \mathcal{O})$ aux places archimédiennes et de 1 aux autres places. Le plongement $\iota$ envoie un sous-espace vectoriel de $E$ de dimension $n^{\prime}$ vers un sous-espace de $\mathfrak{E}$ de dimension $n^{\prime} D$. Par conséquent, il y a au moins $\ell+1$ vecteurs parmi les $u_{i}$ qui sont $k$-linéairement indépendants et donc

$$
\lambda_{\ell+1}(\bar{E}) \leq\left|r \lambda_{\mathbf{A}}\right|_{\mathbf{A}}=|r|_{\mathbf{A}} \lambda_{\ell D+1}(C, \mathcal{O})^{D} .
$$

Cet argument montre aussi que l'estimation (12) reste vraie lorsque l'on remplace $\lambda_{\ell+1}(\bar{E})$ par $\lambda_{\ell+1}^{\mathrm{BV}}(\bar{E})$ à condition que $r$ soit un idèle de Bombieri-Vaaler. Une fois le lien entre $\lambda_{\ell+1}(\bar{E})$ et $\lambda_{\ell D+1}(C, \mathcal{O})$ établi, on utilise une version un peu plus faible du théorème 1.5 de [7] qui affirme que

$$
\operatorname{card}(C \cap \iota(\mathcal{O}))<2^{2 n D-1} \prod_{i=1}^{n D}\left(1+\frac{1}{\lambda_{i}(C, \mathcal{O})}\right)
$$

*I.e. de la forme $(\lambda, \ldots, \lambda, 1,1, \ldots)$ avec $\lambda \in] 0,+\infty[$, voir $\S 2.4$. 
(l'hypothèse $n D \geq 2$ qui se trouve dans l'énoncé original de [7] n'est plus nécessaire). La croissance de la fonction $i \mapsto \lambda_{i}(C, \mathcal{O})$ entraîne

$$
\operatorname{card}(C \cap \iota(\mathcal{O}))<2^{2 n D-1} \prod_{\ell=0}^{n-1}\left(1+\frac{1}{\lambda_{\ell D+1}(C, \mathcal{O})}\right)^{D}
$$

puis, par convexité de $x \mapsto x^{D}$, on a

$$
\operatorname{card}(C \cap \iota(\mathcal{O}))<2^{2 n D-1} \prod_{\ell=0}^{n-1}\left\{2^{D-1}\left(1+\frac{1}{\lambda_{\ell D+1}(C, \mathcal{O})^{D}}\right)\right\}
$$

et le théorème 2.19 se déduit de la majoration (12).

2.5.1. Deuxième théorème de Blichfeldt. Le théorème 2.19 donne lieu à une variante du fameux théorème de Blichfeldt [1] : Si $C$ est un corps convexe de l'espace euclidien $\mathbf{R}^{n}$ contenant 0 et tel que $C \cap \mathbf{Z}^{n}$ n'est pas inclus dans un hyperplan alors $\operatorname{card}\left(C \cap \mathbf{Z}^{n}\right) \leq n ! \operatorname{vol}(C)+n$, sous la forme suivante : si $|r|_{\mathbf{A}} \geq \lambda_{m}(\bar{F})$ alors on a

$$
\operatorname{card}\left(F \cap \mathbf{B}_{\bar{E}}(0, r)\right)<(5 m)^{m D} \delta(\bar{F})^{m} \mu_{F}\left(\mathbf{B}_{\bar{F}}(0, r)\right)
$$

(voir le $\S 2.2$ pour la définition précise de $\mu_{F}$ ). En effet, lorsque $|r|_{\mathbf{A}} \geq \lambda_{m}(\bar{F})$, le théorème 2.19 et la proposition 2.18 fournissent l'estimation

$$
\operatorname{card}\left(F \cap \mathbf{B}_{\bar{E}}(0, r)\right)<\frac{2^{3 m D-1}|r|_{\mathbf{A}}^{m}}{\lambda_{1}(\bar{F}) \cdots \lambda_{m}(\bar{F})}<\frac{\left(8^{D}|r|_{\mathbf{A}} \Delta(\bar{F}) \delta(\bar{F})\right)^{m}}{H(\bar{F})} .
$$

La définition de $H(\bar{F})$ et l'estimation uniforme $\Delta(\bar{F}) \leq(2 m)^{D / 2}$ (inégalité $(6)$ ) conduisent à une majoration en termes du volume de la boule $\mathbf{B}_{\bar{F}}(0, r)$ :

$$
\operatorname{card}\left(F \cap \mathbf{B}_{\bar{E}}(0, r)\right)<\frac{\left(8(2 m)^{1 / 2}\right)^{m D} \delta(\bar{F})^{m}}{2^{r_{2} m} V_{m}^{r_{1}} V_{2 m}^{r_{2}}} \mu_{F}\left(\mathbf{B}_{\bar{F}}(0, r)\right)
$$

( $V_{m}$ est le volume de la boule euclidienne de rayon 1 pour la mesure de Lebesgue usuelle sur $\mathbf{R}^{n}$ ). La majoration (13) découle alors de l'égalité $\frac{1}{V_{m}}=\frac{\Gamma\left(1+\frac{m}{2}\right)}{\pi^{m / 2}}$ et du lemme suivant.

Lemme 2.20. Pour tout nombre réel $x \geq 1$ on a $\Gamma(1+x) \leq x^{x}$.

Démonstration. Au moyen de la convexité de la fonction $\Gamma$, il est facile de voir que, pour tout $x \geq 1$, on a $\Gamma(x) \leq \Gamma(1+[x])([x]$ est la partie entière de $x)$. Ainsi $\Gamma(1+x)=x \Gamma(x) \leq x[x] ! \leq x\left(x^{x-1}\right)=x^{x}$.

2.5.2. Second théorème de Minkowski. En mettant en relation la majoration

$$
\operatorname{card}\left(E \cap \mathbf{B}_{\bar{E}}(0, r)\right)<\frac{2^{3 n D-1}|r|_{\mathbf{A}}^{n}}{\lambda_{1}^{\mathrm{BV}}(\bar{E}) \cdots \lambda_{n}^{\mathrm{BV}}(\bar{E})},
$$

valide lorsque $r$ est un idèle de Bombieri-Vaaler de module plus grand que $\lambda_{n}^{\mathrm{BV}}(\bar{E})$ (majoration déduite du théorème 2.19 comme pour (14)), et la minoration de van der Corput (corollaire 2.12), on obtient, après simplification par $|r|_{\mathbf{A}}^{n}$,

$$
\frac{\operatorname{vol}\left(\mathbf{B}_{\bar{E}}(0,1)\right)}{2^{n D} \operatorname{covol}(E)} \leq \frac{2^{3 n D-1}}{\lambda_{1}^{\mathrm{BV}}(\bar{E}) \cdots \lambda_{n}^{\mathrm{BV}}(\bar{E})} .
$$

Cette inégalité n'est rien d'autre que la variante adélique du second théorème de Minkowski [2], légèrement affaiblie par la présence du terme $2^{3 n D-1}$. 
2.5.3. Avant de conclure ce paragraphe, nous allons obtenir une version plus maniable du théorème 2.19 . Si $x_{m} \leq \cdots \leq x_{1}$ sont des nombres réels positifs, le produit $\prod_{i=1}^{m}\left(1+x_{i}\right)$ est la somme de $x_{1} \cdots x_{m}$ et de $2^{m}-1$ autres termes tous plus petits que $\max \left\{1, x_{1}\right\}^{m-1}$. En particulier on a

$$
\prod_{i=1}^{m}\left(1+x_{i}\right) \leq 2^{m}\left(1+x_{1}^{m-1}+x_{1} \cdots x_{m}\right) \text {. }
$$

En choisissant $x_{i}=|r|_{\mathbf{A}} / \lambda_{i}(\bar{F})$ découle du théorème 2.19 et de la proposition 2.18 l'énoncé suivant.

Proposition 2.21. Soit $\bar{F} \subseteq \bar{E}$ un sous-fibré vectoriel adélique de $\bar{E}$ sur Spec $k$, de dimension $m \geq 1$. Alors, pour tout $r \in k_{\mathbf{A}}$, on a

$$
\operatorname{card}\left(F \cap \mathbf{B}_{\bar{E}}(0, r)\right)<2^{3 m D-1}\left(1+\frac{|r|_{\mathbf{A}}^{m}(\Delta(\bar{F}) \delta(\bar{F}))^{m}}{H(\bar{F})}+\left(\frac{|r|_{\mathbf{A}}}{\lambda_{1}(\bar{F})}\right)^{m-1}\right)
$$

\section{Lemme De Siegel Uniforme}

Le résultat principal de ce paragraphe m'a été communiqué par Gaël Rémond. En substance, l'argumentation qui va suivre est contenue dans les pages 115 à 117 de $[8]$.

Soit $k$ un corps global et $\bar{E}$ un fibré vectoriel adélique sur Spec $k$, de dimension $n \geq 1$. Soit $M \in \mathbf{N} \backslash\{0\}$ et $F, E_{1}, \ldots, E_{M}$ des sous-espaces vectoriels de $E$, de dimensions respectives $m, n_{1}, \ldots, n_{M}$. Avant de passer à la démonstration du théorème 1.1 et de ses variantes, il peut être utile de remarquer que l'on dispose d'une estimation simple et uniforme d'un petit vecteur de $F \backslash \bigcup_{i=1}^{M} E_{i}$. Pour tout $i \in\{1, \ldots, M\}$, considérons $\ell_{1, i}, \ldots, \ell_{n-n_{i}, i}$ des formes linéaires sur $E$ dont l'intersection des noyaux est $E_{i}$. Soit $s=\max \left\{n_{1}, \ldots, n_{M}\right\}$, entier que l'on suppose strictement plus petit que $m$ (comme dans l'énoncé du théorème 1.1). Soit $r=\left(r_{v}\right)_{v} \in k_{\mathbf{A}}$ tel que $F \cap \mathbf{B}_{\bar{E}}(0, r)$ contient une famille libre $\mathcal{F}=\left\{f_{1}, \ldots, f_{s+1}\right\}$. Pour tout $i \in\{1, \ldots, M\}$, comme $\mathcal{F} \not \subset E_{i}\left(\operatorname{car} n_{i}<s+1\right)$, il existe $\sigma(i) \in\left\{1, \ldots, n-n_{i}\right\}$ tel que $\ell_{\sigma(i), i}$ n'est pas identiquement nulle sur $\mathcal{F}$. Le polynôme $P$ de $k\left[X_{1}, \ldots, X_{s+1}\right]$ défini par

$$
P\left(X_{1}, \ldots, X_{s+1}\right)=\prod_{i=1}^{M}\left(\sum_{h=1}^{s+1} X_{h} \ell_{\sigma(i), i}\left(f_{h}\right)\right)
$$

n'est pas identiquement nul sinon l'intégrité de $k\left[X_{1}, \ldots, X_{s+1}\right]$ contredirait l'existence d'au moins un $\sigma(i)$. Son degré partiel par rapport à chacune des variables est inférieur à $M$. Par conséquent, il existe $\left(x_{1}, \ldots, x_{s+1}\right) \in([-M, M] \cap \mathbf{Z} \backslash\{0\})^{s+1}$ tel que $P\left(x_{1}, \ldots, x_{s+1}\right) \neq 0$. Cela signifie que le vecteur $x:=\sum_{h=1}^{s+1} x_{h} f_{h}$ appartient à $F \backslash \bigcup_{i=1}^{M} E_{i}$. De plus, pour toute place $v$ de $k$, on a $\|x\|_{\bar{E}, v} \leq((s+1) M)^{\varepsilon_{v}}\left|r_{v}\right|_{v}$ avec $\varepsilon_{v}=1$ si $v$ est archimédienne et $\varepsilon_{v}=0$ sinon. De là découle l'énoncé suivant.

Proposition 3.1 (G. Rémond). Soit $\bar{E}, F, E_{1}, \ldots, E_{M}$ comme ci-dessus. Supposons que $s=\max \left\{n_{1}, \ldots, n_{M}\right\}<m$. Alors il existe $x \in F \backslash \bigcup_{i=1}^{M} E_{i}$ tel que $H_{\bar{E}}(x) \leq$ $((s+1) M)^{D \delta} \lambda_{s+1}(\bar{F})$.

Remarque 3.2. En remplaçant les formes linéaires par des polynômes, on pourrait obtenir un énoncé plus général avec un ensemble algébrique de degré $M$ ne contenant pas $F$, à la place de $\bigcup_{i=1}^{M} E_{i}$.

La variante adélique du second théorème de Minkowski permet d'évaluer $\lambda_{s+1}(\bar{F})$ qui apparaît dans cette proposition. En effet, dans ce cas, on a

$$
\lambda_{1}(\bar{F}) \cdots \lambda_{m}(\bar{F}) \leq \kappa^{m} H(\bar{F}) \delta(\bar{F})^{m(1-\delta)}
$$


avec

$$
\kappa:= \begin{cases}\frac{2^{r_{1}+r_{2}}\left|D_{k}\right|^{1 / 2}}{V_{m}^{r_{1} / m} V_{2 m}^{r_{2} / m}} & \text { si } k \text { est un corps de nombres, } \\ q^{2 g(k)} & \text { si } k \text { est un corps de fonctions. }\end{cases}
$$

Cela résulte des travaux de Bombieri \& Vaaler [2] pour les corps de nombres et de Thunder [10] pour les corps de fonctions. Dans ce dernier cas, il s'agit plus précisément du corollaire 1 de [10], qui est écrit avec $\Lambda_{i}(\bar{F})$ et $\bar{F}$ pur. Le cas général présenté ici découle de la proposition 2.16. Le défaut de pureté $\delta(\bar{F})$ n'apparaît que dans le cas d'un corps de fonctions car le théorème de Thunder ne porte que sur les fibrés adéliques purs. L'estimation triviale

$$
\lambda_{1}(\bar{F})^{s} \lambda_{s+1}(\bar{F})^{m-s} \leq \lambda_{1}(\bar{F}) \cdots \lambda_{m}(\bar{F})
$$

entraîne alors l'énoncé suivant, qui, outre l'uniformité vis-à-vis des espaces $E_{i}$, a aussi l'élégance de s'appliquer pour un corps de fonctions, contrairement au théorème 1.1 .

Corollaire 3.3. Soit $\bar{E}, F, E_{1}, \ldots, E_{M}$ comme ci-dessus. Supposons que $s=\max \left\{n_{1}, \ldots, n_{M}\right\}<$ m. Alors il existe $x \in F \backslash \bigcup_{i=1}^{M} E_{i}$ tel que

$$
H_{\bar{E}}(x) \leq((s+1) M)^{D \delta}\left(\frac{\kappa^{m} H(\bar{F}) \delta(\bar{F})^{m(1-\delta)}}{\lambda_{1}(\bar{F})^{s}}\right)^{\frac{1}{m-s}} .
$$

Les dépendances en le discriminant du corps de nombres et en la hauteur de $\bar{F}$ sont aussi bonnes que dans le théorème 1.1, dont la démonstration est l'objet du paragraphe suivant.

\section{LE THÉORÈME PRINCIPAL ET SA DÉMONSTRATION}

Voici le résultat principal de cet article qui généralise le théorème 1.1, présenté dans l'introduction.

Théorème 4.1. Soit $k$ un corps de nombres de degré $D$ et de discriminant absolu $D_{k}$. Soit $\bar{E}$ un fibré vectoriel adélique sur $\operatorname{Spec} k$ et $\bar{F} \subseteq \bar{E}$ un sous-fibré vectoriel adélique, de dimension $m$. Soit $M \in \mathbf{N} \backslash\{0\}$ et $E_{1}, \ldots, E_{M}$ des sous-espaces vectoriels de $E$ de dimensions respectives $n_{1}, \ldots, n_{M}$. Posons

$$
H:=(41 m)^{D / 2}\left|D_{k}\right|^{1 / 2}(M H(\bar{F}))^{1 / m}
$$

et

$$
R:=H \max _{1 \leq i \leq M}\left\{1,\left(\frac{H^{n_{i}}\left(\Delta\left(\overline{E_{i}}\right) \delta\left(\overline{E_{i}}\right)\right)^{n_{i}}}{H\left(\overline{E_{i}}\right)}\right)^{\frac{1}{m-n_{i}}},\left(\frac{H}{\lambda_{1}\left(\overline{E_{i}}\right)}\right)^{\frac{n_{i}-1}{m-n_{i}+1}}\right\} .
$$

Supposons que $\max \left\{n_{1}, \ldots, n_{M}\right\}<m$. Alors, pour tout $r=\left(r_{v}\right)_{v} \in k_{\mathbf{A}}^{\times}$de module supérieur à $R$, il existe $x \in F \backslash \bigcup_{i=1}^{M} E_{i}$ tel que, pour toute place $v$ de $k$, on a $\|x\|_{\bar{E}, v} \leq\left|r_{v}\right|_{v}$.

En particulier, l'on peut trouver $x \in F \backslash \bigcup_{i=1}^{M} E_{i}$ tel que $H_{\bar{E}}(x) \leq R$.

Démonstration. L'existence du vecteur $x$ repose sur la capacité de choisir $r \in k_{\mathbf{A}}$ de valeur absolue $|r|_{\text {A }}$ aussi petite que possible de sorte que l'on ait

$$
\sum_{i=1}^{M}\left(\operatorname{card}\left(E_{i} \cap \mathbf{B}_{\bar{E}}(0, r)\right)-1\right)<\operatorname{card}\left(F \cap \mathbf{B}_{\bar{E}}(0, r)\right)-1
$$

(le -1 correspond au vecteur nul que l'on ne veut pas compter $M$ fois). Pour un tel $r$, qui est nécessairement un idèle, il existe un vecteur $x$ de $F$, qui n'appartient à aucun $\operatorname{des} E_{i}, i \in\{1, \ldots, M\}$, tel que, pour toute place $v$ de $k$, on a $\|x\|_{\bar{E}, v} \leq\left|r_{v}\right|_{v}$. Si tous 
les $E_{i}$ sont nuls, le membre de gauche de (16) est nul. Dans ce cas, pour satisfaire la condition (16), on fait appel au corollaire 2.12 qui minore $\operatorname{card}\left(F \cap \mathbf{B}_{\bar{E}}(0, r)\right)$ et à la formule (9) pour affirmer que si $|r|_{\mathbf{A}}$ est plus grand que

$$
2^{D}\left(\frac{\operatorname{covol}(F)}{\operatorname{vol}\left(\mathbf{B}_{\bar{F}}(0,1)\right)}\right)^{1 / m}=2^{D}\left(\frac{H(\bar{F})\left|D_{k}\right|^{m / 2}}{2^{m r_{2}} V_{m}^{r_{1}} V_{2 m}^{r_{2}}}\right)^{1 / m}
$$

( $V_{m}$ désigne le volume de la boule euclidienne de $\mathbf{R}^{m}$ ) alors le théorème est démontré avec $|r|_{\mathbf{A}}$ au lieu de $R$. Le lemme 2.20 permet alors de conclure en montrant que la quantité (17) est plus petite que $R$. Si l'un des $E_{j}$ n'est pas nul, on peut supposer qu'aucun de ces espaces n'est nul, quitte à supprimer au départ et dans (16) les entiers $i$ pour lesquels $E_{i}=\{0\}$ (le nombre $M$ de sous-espaces décroît et le théorème 4.1 n'en sera que meilleur). En particulier, on peut supposer maintenant que $m \geq 2$. Compte tenu de la proposition 2.21 qui majore $\operatorname{card}\left(E_{i} \cap \mathbf{B}_{\bar{E}}(0, r)\right)$ et du corollaire 2.12 qui minore card $\left(F \cap \mathbf{B}_{\bar{E}}(0, r)\right)$, il suffit alors de choisir $r$ de sorte que $|r|_{\text {A }}$ vérifie l'inéquation

(18)

$$
\sum_{i=1}^{M} 2^{3 n_{i} D-1}\left(1+\frac{|r|_{\mathbf{A}}^{n_{i}}\left(\Delta\left(\overline{E_{i}}\right) \delta\left(\overline{E_{i}}\right)\right)^{n_{i}}}{H\left(\overline{E_{i}}\right)}+\left(\frac{|r|_{\mathbf{A}}}{\lambda_{1}\left(\overline{E_{i}}\right)}\right)^{n_{i}-1}\right) \leq \frac{|r|_{\mathbf{A}}^{m} \operatorname{vol}\left(\mathbf{B}_{\bar{F}}(0,1)\right)}{2^{m D} \operatorname{covol}(F)}
$$

Pour qu'une inégalité de la forme $x_{1}+\ldots+x_{3 M} \leq x$ soit satisfaite, il suffit que $x_{i} \leq$ $x /(3 M)$ pour tout $i \in\{1, \ldots, 3 M\}$. Ce principe appliqué à (18) et la majoration $n_{i} \leq m-1$ conduisent à requérir pour $|r|_{\mathbf{A}}$ les conditions suivantes :

(i) $\left(\frac{16^{m D} M \operatorname{covol}(F)}{\operatorname{vol}\left(\mathbf{B}_{\bar{F}}(0,1)\right)}\right)^{1 / m} \leq|r|_{\mathbf{A}}$

(ii) Pour tout $i \in\{1, \ldots, M\},\left(\frac{16^{m D} M \operatorname{covol}(F)\left(\Delta\left(\overline{E_{i}}\right) \delta\left(\overline{E_{i}}\right)\right)^{n_{i}}}{\operatorname{vol}\left(\mathbf{B}_{\bar{F}}(0,1)\right) H\left(\overline{E_{i}}\right)}\right)^{1 /\left(m-n_{i}\right)} \leq|r|_{\mathbf{A}}$

(iii) Pour tout $i \in\{1, \ldots, M\},\left(\frac{16^{m D} M \operatorname{covol}(F)}{\operatorname{vol}\left(\mathbf{B}_{\bar{F}}(0,1)\right) \lambda_{1}\left(\overline{E_{i}}\right)^{n_{i}-1}}\right)^{1 /\left(m-n_{i}+1\right)} \leq|r|_{\mathbf{A}}$

Comme précédemment, on utilise la formule $(9)$ pour évaluer le quotient $\operatorname{covol}(F) / \operatorname{vol}\left(\mathbf{B}_{\bar{F}}(0,1)\right)$ et on observe que, comme $m \geq 2$, on a

$$
\frac{16^{m D}}{2^{m r_{2}} V_{m}^{r_{1}} V_{2 m}^{r_{2}}} \leq(41 m)^{m D / 2}
$$

grâce au lemme 2.20 .

\section{VARIANTES DU THÉORÈME PRINCIPAL}

Le théorème 4.1 est le résultat d'un compromis entre le théorème 2.19 et la proposition 2.21 qui en est une version affaiblie mais plus maniable. D'autres arbitrages conduisent à des variantes du théorème 4.1, que nous présentons ici.

Théorème 5.1. Soit $k$ un corps de nombres et $\bar{E}$ un fibré vectoriel adélique sur Spec $k$. Soit $\bar{F} \subseteq \bar{E}$ un sous-fibré vectoriel adélique, de dimension $m$. Soit $M \in$ $\mathbf{N} \backslash\{0\}$ et $E_{1}, \ldots, E_{M}$ des sous-espaces vectoriels de $E$ de dimensions respectives $n_{1}, \ldots, n_{M}$. Notons

$$
H=(41 m)^{D / 2}\left|D_{k}\right|^{1 / 2}(M H(\bar{F}))^{1 / m} .
$$

Supposons que $\max \left\{n_{1}, \ldots, n_{M}\right\}<m$. Alors il existe $x \in F \backslash \bigcup_{i=1}^{M} E_{i}$ tel que

$$
H_{\bar{E}}(x) \leq H \max _{1 \leq i \leq M}\left\{1,\left(\frac{H}{\lambda_{1}\left(\overline{E_{i}}\right)}\right)^{\frac{n_{i}}{m-n_{i}}}\right\} \text {. }
$$


Démonstration. La démonstration est identique à celle du théorème 4.1 à ceci près que la majoration de $\operatorname{card}\left(E_{i} \cap \mathbf{B}_{\bar{E}}(0, r)\right)$ n'est pas celle de la proposition 2.21. On utilise plutôt

$$
\operatorname{card}\left(E_{i} \cap \mathbf{B}_{\bar{E}}(0, r)\right)<2^{3 n_{i} D-2}\left(1+\left(\frac{|r|_{\mathbf{A}}}{\lambda_{1}\left(\overline{E_{i}}\right)}\right)^{n_{i}}\right),
$$

qui découle directement du théorème 2.19 en minorant $\lambda_{j}\left(\overline{E_{i}}\right)$ par $\lambda_{1}\left(\overline{E_{i}}\right)$, pour $j \in\left\{1, \ldots, n_{i}\right\}$, et en utilisant $(1+x)^{n_{i}} \leq 2^{n_{i}-1}\left(1+x^{n_{i}}\right)$ pour $x \in \mathbf{R}$. Le même raisonnement que celui qui suit l'inégalité (18) conduit à demander que $|r|_{\mathbf{A}}$ satisfasse aux deux conditions :

(i) $\left(\frac{16^{m D} M \operatorname{covol}(F)}{\operatorname{vol}\left(\mathbf{B}_{\bar{F}}(0,1)\right)}\right)^{1 / m} \leq|r|_{\mathbf{A}}$

(ii) Pour tout $i \in\{1, \ldots, M\},\left(\frac{16^{m D} M \operatorname{covol}(F)}{\operatorname{vol}\left(\mathbf{B}_{\bar{F}}(0,1)\right) \lambda_{1}\left(\overline{E_{i}}\right)^{n_{i}}}\right)^{1 /\left(m-n_{i}\right)} \leq|r|_{\mathbf{A}}$

Le théorème 5.1 s'obtient alors exactement comme à la fin du paragraphe précédent.

Dans la théorie des fibrés vectoriels adéliques, on désigne habituellement par $\widehat{\mu}_{\max }(\bar{E})$ le maximum des nombres réels $-\frac{\log H\left(\overline{E^{\prime}}\right)}{[k: \mathbf{Q}] \operatorname{dim}\left(E^{\prime}\right)}$ lorsque $E^{\prime}$ parcourt les sousespaces vectoriels non nul de $E$ (voir [6] pour l'existence de ce maximum). Pour un tel $E^{\prime}$, il existe $e^{\prime} \in E^{\prime}$ tel que $\Lambda_{1}\left(\overline{E^{\prime}}\right)=H_{\bar{E}}\left(e^{\prime}\right)$. Cette quantité est aussi la hauteur du sous-espace $k . e^{\prime}$ de $E$. On a donc

$$
\lambda_{1}\left(\overline{E^{\prime}}\right) \geq \Lambda_{1}\left(\overline{E^{\prime}}\right) \geq e^{-D \widehat{\mu}_{\max }(\bar{E})}
$$

pour tout sous-espace $\{0\} \neq E^{\prime} \subseteq E$. Le théorème 5.1 conduit alors à la borne uniforme suivante.

Corollaire 5.2. Reprenons les notations et hypothèses du théorème 5.1. Soit $s=$ $\max \left\{n_{1}, \ldots, n_{M}\right\}$. Alors il existe $x \in F \backslash \bigcup_{i=1}^{M} E_{i}$ tel que

$$
H_{\bar{E}}(x) \leq\left(H^{m} e^{s D \widehat{\mu}_{\max }(\bar{F})}\right)^{\frac{1}{m-s}} .
$$

Démonstration. On utilise le théorème 5.1 avec $E_{i} \cap F$ à la place de $E_{i}$ et l'on minore $\lambda_{1}\left(\overline{E_{i} \cap F}\right)$ par $e^{-D \widehat{\mu}_{\max }(\bar{F})}$. Le corollaire découle alors de la minoration $H(\bar{F})^{\frac{1}{m}} e^{D \widehat{\mu}_{\max }(\bar{F})} \geq 1$ et de la croissance de la fonction $x \mapsto \frac{x}{m-x}$ pour $x \in[0, m-$ $1]$.

Voici une autre variante du théorème 4.1.

Théorème 5.3. Reprenons les notations et hypothèses du théorème 5.1. Alors il existe $x \in F \backslash \bigcup_{i=1}^{M} E_{i}$ tel que

$$
H_{\bar{E}}(x) \leq H \max _{1 \leq i \leq M}\left\{\frac{\lambda_{n_{i}}\left(\overline{E_{i}}\right)}{H},\left(\frac{H^{n_{i}}\left(\Delta\left(\overline{E_{i}}\right) \delta\left(\overline{E_{i}}\right)\right)^{n_{i}}}{H\left(\overline{E_{i}}\right)}\right)^{\frac{1}{m-n_{i}}}\right\} .
$$

Démonstration. Là encore la démonstration est presque celle du théorème 4.1. Observons que, pour $|r|_{\mathbf{A}} \geq \lambda_{n_{i}}\left(\overline{E_{i}}\right)$, le théorème 2.19 et la proposition 2.18 impliquent

$$
\operatorname{card}\left(E_{i} \cap \mathbf{B}_{\bar{E}}(0, r)\right) \leq \frac{2^{3 n_{i} D-1}|r|_{\mathbf{A}}^{n_{i}}}{\lambda_{1}\left(\overline{E_{i}}\right) \cdots \lambda_{n_{i}}\left(\overline{E_{i}}\right)} \leq \frac{2^{3 n_{i} D-1}|r|_{\mathbf{A}}^{n_{i}}\left(\Delta\left(\overline{E_{i}}\right) \delta\left(\overline{E_{i}}\right)\right)^{n_{i}}}{H\left(\overline{E_{i}}\right)}
$$

Ainsi la condition $\sum_{i=1}^{M} \operatorname{card}\left(E_{i} \cap \mathbf{B}_{\bar{E}}(0, r)\right)<\operatorname{card}\left(F \cap \mathbf{B}_{\bar{E}}(0, r)\right)$, qui garantit l'existence d'un $x \in F \backslash \bigcup_{i=1}^{M} E_{i}$ de hauteur $\leq|r|_{\mathbf{A}}$, est satisfaite dès lors que

(i) $\max \left\{\lambda_{n_{1}}\left(\overline{E_{1}}\right), \ldots, \lambda_{n_{M}}\left(\overline{E_{M}}\right)\right\} \leq|r|_{\mathbf{A}}$ et

(ii) pour tout $i \in\{1, \ldots, M\},\left(\frac{16^{m D} M \operatorname{covol}(F)\left(\Delta\left(\overline{E_{i}}\right) \delta\left(\overline{E_{i}}\right)\right)^{n_{i}}}{\operatorname{vol}\left(\mathbf{B}_{\bar{F}}(0,1)\right) H\left(\overline{E_{i}}\right)}\right)^{1 /\left(m-n_{i}\right)} \leq|r|_{\mathbf{A}}$ 
(on utilise le corollaire 2.12 et la majoration $n_{i} \leq m-1$ ). Cette deuxième condition est exactement la condition (ii) qui intervient dans la démonstration du théorème 4.1. Par conséquent les calculs faits à cette occasion restent valides et le théorème 5.3 s'ensuit.

\section{Liens AVEC la GÉOMÉtrie Des nOMBRES ClASSique}

Ce dernier paragraphe traduit une partie des résultats que nous venons de présenter dans le langage de la géométrie des nombres classique.

Soit $n$ un entier $\geq 1$ et $C \subseteq \mathbf{R}^{n}$ une partie convexe, d'intérieur non vide, compacte et symétrique par rapport à l'origine. Cet ensemble est la boule unité fermée de $\mathbf{R}^{n}$ muni de la norme jauge:

$$
\forall x \in \mathbf{R}^{n}, \quad\|x\|_{C}:=\inf \left\{\lambda>0 ; \frac{x}{\lambda} \in C\right\} .
$$

Soit $\Omega$ un réseau complet de $\mathbf{R}^{n}$, i.e. un sous-groupe discret de $\operatorname{rang} n$ de $\mathbf{R}^{n}$. Il existe une base $\left(\omega_{1}, \ldots, \omega_{n}\right)$ de $\mathbf{R}^{n}$ telle que $\Omega=\bigoplus_{i=1}^{n} \mathbf{Z} . \omega_{i}$. Le Q-espace vectoriel $\Omega \otimes_{\mathbf{Z}} \mathbf{Q}$ peut alors être muni d'une collection de normes $\left(\|\cdot\|_{v}\right)_{v}$, indexée par les places $v$ de $\mathbf{Q}$, de la manière suivante :

- Si $v$ est la place archimédienne $v_{0}$ de $\mathbf{Q}$ alors, pour tout $x=a+i b \in \Omega \otimes_{\mathbf{Z}} \mathbf{C}$ $\left(a, b \in \mathbf{R}^{n}\right)$, on a $\|x\|_{v_{0}}:=\left(\|a\|_{C}^{2}+\|b\|_{C}^{2}\right)^{1 / 2}\left(^{*}\right)$,

- si $v$ est ultramétrique alors, pour tout $\left(x_{1}, \ldots, x_{n}\right) \in \mathbf{C}_{v}^{n}$, on a

$$
\left\|\sum_{i=1}^{n} x_{i} \omega_{i}\right\|_{v}:=\max \left\{\left|x_{1}\right|_{v}, \ldots,\left|x_{n}\right|_{v}\right\} .
$$

Les normes aux places ultramétriques ne dépendent pas du choix de la Z-base $\left(\omega_{1}, \ldots, \omega_{n}\right)$ de $\Omega$ car une matrice de $\mathrm{GL}_{n}(\mathbf{Z})$ est une isométrie pour la norme du maximum ultramétrique. Sa structure entière est donnée par $\Omega$, i.e.

$$
\Omega=\left\{x \in \Omega \otimes_{\mathbf{z}} \mathbf{Q} ; \quad \forall v \neq v_{0}, \quad\|x\|_{v} \leq 1\right\} .
$$

Avec un léger abus d'écriture, on notera $\left(\Omega,\|\cdot\|_{C}\right)$ le fibré vectoriel adélique pur ainsi construit. Il faut prendre garde que si $\mho$ est un sous-groupe de $\Omega$, le fibré adélique $\left(\mho,\|\cdot\|_{C}\right)$ n'est pas a priori un sous-fibré adélique de $\left(\Omega,\|\cdot\|_{C}\right)$. La raison en est que les structures entières ne coïncident pas en général. Pour que cela soit le cas, il faut et il suffit que $\mho$ soit égal à $\left(\mho \otimes_{\mathbf{z}} \mathbf{Q}\right) \cap \Omega$ i.e. que le quotient $\Omega / \mho$ soit sans torsion, ou, ce qui revient au même, que $\mho$ soit facteur direct dans $\Omega$ (on dit alors que $\mho$ est saturé).

Dans ce contexte, il est possible de calculer la hauteur de $\left(\Omega,\|\cdot\|_{C}\right)$ au sens du $\S 2.2$. Rappelons que $V_{n}$ désigne le volume de la boule euclidienne de $\mathbf{R}^{n}$ mesuré par rapport à la mesure de Lebesgue usuelle. On a alors

$$
H\left(\Omega,\|\cdot\|_{C}\right)=\frac{V_{n} \operatorname{covol}(\Omega)}{\operatorname{vol}(C)}
$$

où vol est une mesure de Haar sur $\mathbf{R}^{n}$ et $\operatorname{covol}(\Omega)$ est la mesure du quotient $\mathbf{R}^{n} / \Omega$ induite par vol. Si vol est la mesure de Lebesgue usuelle sur $\mathbf{R}^{n}$ alors $\operatorname{covol}(\Omega)=$ $\left|\operatorname{det}\left(\omega_{1}, \ldots, \omega_{n}\right)\right|$ pour toute $\mathbf{Z}$-base $\left(\omega_{1}, \ldots, \omega_{n}\right)$ de $\Omega$. Afin d'avoir une formule similaire à (19) mais avec un sous-groupe $\mho$ de $\Omega$, nous allons supposer que $C$ est la boule unité de $\mathbf{R}^{n}$ pour la norme euclidienne $|\cdot|_{2}$. Considérons une $\mathbf{Z}$-base $\mathfrak{u}_{1}, \ldots, \mathfrak{u}_{m}$

${ }^{*}$ En réalité seule la trace de cette norme sur $\Omega \otimes_{\mathbf{Z}} \mathbf{R}$ sera utile (pour calculer le volume de la boule unité adélique par exemple). Aussi aurions-nous pu prendre $\max \left\{\|a\|_{C},\|b\|_{C}\right\}$ ou tout autre choix donnant $\|a\|_{C}$ lorsque $b=0$ et invariante par $b \mapsto-b$. 
de $\mho$. Chaque vecteur $\mathfrak{u}_{j}$ s'écrit comme une somme $\sum_{i=1}^{n} u_{i, j} \omega_{i}$ avec $u_{i, j} \in \mathbf{Z}$. Soit $\mathfrak{U}$ la matrice $\left(u_{i, j}\right)_{\substack{1 \leq i \leq n \\ 1 \leq j \leq m}} \in \mathrm{M}_{n, m}(\mathbf{Z})$. Alors on a

$$
H\left(\left(\mho \otimes_{\mathbf{Z}} \mathbf{Q}\right) \cap \Omega,|\cdot|_{2}\right)=\operatorname{det}\left({ }^{\mathrm{t}} \mathfrak{U} \mathfrak{U}\right) \prod_{v \neq v_{0}} \max _{\mathfrak{U}_{0}}\left|\operatorname{det} \mathfrak{U}_{0}\right|_{v}
$$

où les maxima portent sur les mineurs maximaux $\mathfrak{U}_{0}$ de la matrice $\mathfrak{U}$. On retrouve ainsi la formule classique caractérisant la hauteur en termes des coordonnées grassmaniennes de $\mathfrak{U}$. Le terme $\operatorname{det}\left({ }^{\mathrm{t}} \mathfrak{U} \mathfrak{U}\right)$ est la partie archimédienne $V_{m} / \operatorname{vol}(C \cap(\mho \otimes \mathbf{z}$ $\mathbf{R})$ ) de la hauteur. Le produit sur les places $v \neq v_{0}$ est le covolume de

$$
(\mho \otimes \mathbf{z} \mathbf{Q}) \cap \Omega=\left\{x=\sum_{j=1}^{m} x_{j} \mathfrak{u}_{j} \in \mho \otimes \mathbf{Z} \mathbf{Q} ; \mathfrak{U}\left(\begin{array}{c}
x_{1} \\
\vdots \\
x_{m}
\end{array}\right) \in \mathbf{Z}^{n}\right\} .
$$

On s'en convainc aisément en utilisant l'équivalence de $\mathfrak{U}$ avec une matrice $n \times m$ diagonale composée sur la diagonale de représentants des facteurs invariants de $\mathfrak{U}$. De plus on a

$$
H\left(\mho,\|\cdot\|_{C}\right)=\left[\left(\mho \otimes_{\mathbf{Z}} \mathbf{Q}\right) \cap \Omega: \mho\right] \times H\left(\left(\mho \otimes_{\mathbf{z}} \mathbf{Q}\right) \cap \Omega,\|\cdot\|_{C}\right) .
$$

En revenant au cas général, et pour alléger la notation, nous noterons $H(\mho ; \Omega, C)$ la hauteur du sous-fibré vectoriel adélique $\left(\left(\mho \otimes_{\mathbf{Z}} \mathbf{Q}\right) \cap \Omega,\|\cdot\|_{C}\right)$ de $\left(\Omega,\|\cdot\|_{C}\right)$.

À un couple $(C, \Omega)$ sont traditionnellement associés $n$ minima successifs définis de la manière suivante : pour tout $i \in\{1, \ldots, n\}$, le $i^{\text {ème }}$ minimum $\lambda_{i}(C, \Omega)$ est la borne inférieure des nombres réels $\lambda>0$ pour lesquels le sous-ensemble $\lambda C \cap \Omega$ de $\mathbf{R}^{n}$ possède une famille libre de $i$ vecteurs. Cette définition est cohérente avec les trois types de minima du fibré adélique $\left(\Omega,\|\cdot\|_{C}\right)$, définis au $\S 2.4$ : pour tout $i \in\{1, \ldots, n\}$,

$$
\Lambda_{i}\left(\Omega,\|\cdot\|_{C}\right)=\lambda_{i}\left(\Omega,\|\cdot\|_{C}\right)=\lambda_{i}^{\mathrm{BV}}\left(\Omega,\|\cdot\|_{C}\right)=\lambda_{i}(C, \Omega) .
$$

Les deux premières égalités sont un cas particulier du corollaire 2.17 , dont la démonstration comporte une information supplémentaire : lorsque $\bar{E}$ est pur sur $\operatorname{Spec} \mathbf{Q}$, alors, pour tout $H \in H_{\bar{E}}(E)$, il existe $x \in E$ tel que $\|x\|_{\bar{E}, v_{0}}=H$ et $\|x\|_{\bar{E}, v}=1$ pour toute place $v$ ultramétrique de $\mathbf{Q}$. Appliquée au cas de $\left(\Omega,\|\cdot\|_{C}\right)$, cette remarque permet de constater que, pour tout $x \in \Omega \otimes_{\mathbf{z}} \mathbf{Q}$, il existe $d \in \mathbf{Q}$ tel que $d x \in \Omega$ et $H_{\left(\Omega,\|\cdot\|_{C}\right)}(x)=\|d x\|_{C}$ et de montrer alors la dernière égalité avec $\lambda_{i}(C, \Omega)$.

Ces considérations conduisent à formuler un cas particulier de notre résultat principal 4.1 sous la forme suivante.

Théorème 6.1. Soit $C \subseteq \mathbf{R}^{n}$ un corps convexe, d'intérieur non vide, symétrique par rapport à l'origine et $\Omega$ un réseau complet de $\mathbf{R}^{n}$. Soit $M \in \mathbf{N} \backslash\{0\}$ et $\mho, \mho_{1}, \ldots, \mho_{M}$ des sous-groupes de $\Omega$, de rangs $m, n_{1}, \ldots, n_{M}$ respectivement. Posons

$$
H:=7 m(M H(\mho ; \Omega, C))^{1 / m} .
$$

Supposons que $\max \left\{n_{1}, \ldots, n_{M}\right\}<m$. Alors il existe $x \in\left(\left(\mho \otimes_{\mathbf{z}} \mathbf{Q}\right) \cap \Omega\right) \backslash$ $\bigcup_{i=1}^{M} \mho_{i} \otimes_{\mathbf{Z}} \mathbf{Q}$ tel que

$$
\|x\|_{C} \leq H \max _{1 \leq i \leq M}\left\{1,\left(\frac{H^{n_{i}}}{H\left(\mho_{i} ; \Omega, C\right)}\right)^{\frac{1}{m-n_{i}}},\left(\frac{H}{\lambda_{1}\left(C,\left(\mho_{i} \otimes \mathbf{z} \mathbf{Q}\right) \cap \Omega\right)}\right)^{\frac{n_{i}-1}{m-n_{i}+1}}\right\} .
$$

Nous avons majoré le défaut d'hermitianité $\Delta\left(\mho_{i} \otimes_{\mathbf{z}} \mathbf{Q},\|\cdot\|_{C}\right)$ par $\sqrt{n_{i}} \leq \sqrt{m}$, que nous avons alors déplacé dans $H$, en remplaçant $(41 m)^{1 / 2}$ par $7 m$. 


\section{REMERCIEMENTS}

Je remercie Gaël Rémond pour l'intérêt et les contributions apportés à ce texte (dont, en particulier, le $\S 3$ ). Je remercie également le rapporteur du travail qu'il a accompli, avec promptitude. Par ailleurs, ce texte a bénéficié du soutien du projet ANR Diophante, 06-JCJC-0028, piloté par Lucia Di Vizio.

\section{RÉFÉRENCES}

[1] H.F. Blichfeldt. Notes on geometry of numbers. Announcement to the October meeting of the San Francisco section of the A.M.S. (1920). Bull. Amer. Math. Soc. 27(4) :152-153, 1921.

[2] E. Bombieri et J. VAaler. On Siegel's lemma. Invent. math., 73(1) :11-32, 1983. Avec un addendum : ibid. $75(2): 377,1984$.

[3] C. Christensen et W. Gubler. Der relative Satz von Schanuel. Manuscripta Math., 126(4) :505-525, 2008.

[4] L. Fukshansky. Integral points of small height outside of a hypersurface. Monatsh. Math., $147(1): 25-41,2006$.

[5] L. Fukshansky. Siegel's lemma with additional conditions. J. Number Theory, 120(1) :13-25, 2006.

[6] É. Gaudron. Pentes des fibrés vectoriels adéliques sur un corps global. Rend. Sem. Mat. Univ. Padova, 119 :21-95, 2008.

[7] M. Henk. Successive minima and lattice points. Rend. Circ. Mat. Palermo (2) Suppl., 70(I) :377-384, 2002

[8] G. Rémond. Inégalité de Vojta en dimension supérieure. Ann. Scuola Norm. Sup. Pisa Cl. Sci. (4), 29(1):101-151, 2000.

[9] D. Roy et J.L. Thunder. An absolute Siegel's lemma. J. Reine angew. Math., 476 :1-26, 1996. Addendum et erratum, ibid., 508 :47-51, 1999.

[10] J.L. Thunder. An adelic Minkowski-Hlawka theorem and an application to Siegel's lemma. J. reine angew Math., 475 :167-185, 1996.

[11] J.D. VAaler. The best constant in Siegel's lemma. Monatsh. Math., 140(1) :71-89, 2003.

[12] A. WeIl. Basic number theory. Seconde édition de 1973 publiée dans Classics in Mathematics. Springer-Verlag, Berlin, 1995. 